\title{
Flux domes in superconducting films without edges
}

\author{
John R. Clem \\ Ames Laboratory-DOE and Department of Physics and Astronomy, Iowa State University, Ames Iowa 50011, USA \\ Yasunori Mawatari \\ National Institute of Advanced Industrial Science and Technology (AIST), Tsukuba, Ibaraki 305-8568, Japan
}

(Dated: July 15, 2018)

\begin{abstract}
Domelike magnetic-flux-density distributions previously have been observed experimentally and analyzed theoretically in superconducting films with edges, such as in strips and thin plates. Such flux domes have been explained as arising from a combination of strong geometric barriers and weak bulk pinning. In this paper we predict that, even in films with bulk pinning, flux domes also occur when vortices and antivortices are produced far from the film edges underneath current-carrying wires, coils, or permanent magnets placed above the film. Vortex-antivortex pairs penetrating through the film are generated when the magnetic field parallel to the surface exceeds $H_{c 1}+K_{c}$, where $H_{c 1}$ is the lower critical field and $K_{c}=j_{c} d$ is the critical sheet-current density (the product of the bulk critical current density $j_{c}$ and the film thickness $d$ ). The vortices and antivortices move in opposite directions to locations where they join others to create separated vortex and antivortex flux domes. We consider a simple arrangement of a pair of current-carrying wires carrying current $I_{0}$ in opposite directions and calculate the magnetic-field and current-density distributions as a function of $I_{0}$ both in the bulk-pinning-free case $\left(K_{c}=0\right)$ and in the presence of bulk pinning, characterized by a field-independent critical sheet-current density $\left(K_{c}>0\right)$.

PACS numbers: 74.25.Sv,74.25.Nf,74.78.-w
\end{abstract}

\section{INTRODUCTION}

The hysteretic penetration of magnetic flux into a superconductor has long been a subject of experimental and theoretical interest. One phenomenon that has often been observed in flat samples in an increasing perpendicular applied magnetic field is a domelike magnetic-flux-density distribution centered in the middle of the sample, surrounded by a superconducting fluxfree zone. This phenomenon has been investigated experimentally and interpreted theoretically in both typeI superconductors $1,2,3,4,5,6,7,8,9$ and weak-pinning type-II superconductors 10.11 .12 .13 .14 .15 .16 .17 .18 .19 .20 In these previous investigations, the flux domes have been explained as arising from an energy barrier of geometric origin at the edge of the sample. Once this barrier is overcome, flux tubes or vortices escape from the edge and are driven to the middle by Meissner screening currents, which flow on the sample's surface.

In this paper we consider a type-II superconducting film without edges and predict that when it is subjected to local magnetic fields produced by current-carrying wires above the sample, vortex and antivortex magneticflux domes are produced in the film when the currents in the wires are sufficiently large. When bulk pinning is weak, we predict that the vortex and antivortex domes are separated from each other, but as the bulk pinning increases, the space between the vortex and antivortex domes shrinks to zero.

To investigate these effects in an easily calculable geometry, we consider in Sec.IIa simple model in which the local magnetic fields are produced by a pair of infinitely long straight wires. The resulting two-dimensional ge- ometry allows us to calculate all the magnetic-field and sheet-current distributions analytically. In Sec. IA we discuss the Meissner-state response of the film before any penetration of vortices into the film, and in Sec. II we discuss the distributions produced by vortices and antivortices that have penetrated through the film thickness. We then present the magnetic-field and sheetcurrent distributions associated with the flux domes, both in the absence of bulk pinning [Sec. IC and in the presence of weak [Sec. ПD] and strong [Sec. IE] bulk pinning. In Sec. III] we summarize our results, discuss the generality of the predicted effects in more easily realizable experimental configurations, consider similar phenomena in type-I superconductors, and discuss possible extensions of this work. Calculations of screening effects in the Meissner state are presented in Appendix $\mathrm{A}$, and derivations of the complex field and complex potential are given in Appendix B.

\section{SUPERCONDUCTING FILMS AND LINEAR WIRES}

We consider a simple geometry in which vortex and antivortex flux domes are produced far from the film edges. For simplicity we consider an infinite type-II superconducting film and a pair of infinitely long current-carrying wires, as shown in Fig. 1. A wire, carrying current $I_{1}=I_{0}$ parallel to the $z$ axis, is situated at $(x, y)=\left(0, y_{1}\right)$, where $y_{1}>0$. The return current $I_{2}=-I_{0}$ is carried by a second wire at $(x, y)=\left(0, y_{2}\right)$, where $y_{2}>y_{1}$. The wire radius $r_{w}$ is assumed to be much smaller than either $y_{1}$ or the wire separation $y_{2}-y_{1}$. A superconducting 


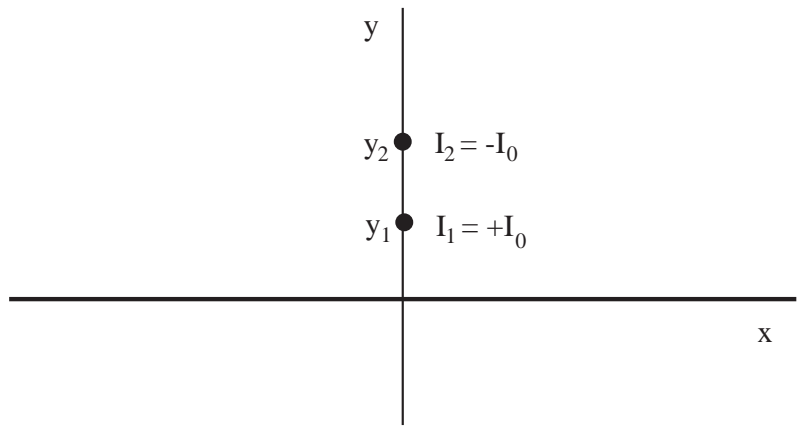

FIG. 1: Wire 1 at $(x, y)=\left(0, y_{1}\right)$ carries current $I_{1}=I_{0}$ in the $z$ direction and wire 2 at $(x, y)=\left(0, y_{2}\right)$ carries current $I_{2}=-I_{0}$ above an infinite type-II superconducting film in the $x z$ plane.

film, infinitely extended in the $x z$ plane, is situated at $-d / 2<y<d / 2$, where the film thickness $d$ is larger than the London penetration depth $\lambda$ and $d \ll y_{1}$. As discussed in Appendix A, these conditions guarantee that when the film is in the Meissner state, the magnetic field below the film is negligibly small.

Flux pinning in the film is characterized by the critical current density $j_{c}$, which is assumed to be constant (independent of magnetic field), as in Bean's critical state model,${ }^{21}$ and isotropic (independent of vortex direction) However, the relevant physical quantity here is the critical sheet-current density $K_{c}=j_{c} d$. Since we are interested in the case for which $d \ll y_{1}$, in the following we ignore the finite thickness of the film, as this simplification allows us to obtain simple analytic expressions for the magnetic-field and current-density distributions.

We introduce the complex field $\mathcal{H}(\zeta)=H_{y}(x, y)+$ $i H_{x}(x, y)$, which is an analytic function of $\zeta=x+i y$ except for poles at $\zeta=\zeta_{1}=i y_{1}$ and $\zeta=\zeta_{2}=i y_{2}$ and a branch cut at $y=0$. The Biot-Savart law for the complex field is given by

$$
\mathcal{H}(\zeta)=\mathcal{H}_{0}(\zeta)+\frac{1}{2 \pi} \int_{-\infty}^{+\infty} d u \frac{K_{z}(u)}{\zeta-u}
$$

where

$$
\mathcal{H}_{0}(\zeta)=\frac{I_{0}}{2 \pi} \frac{1}{\zeta-i y_{1}}-\frac{I_{0}}{2 \pi} \frac{1}{\zeta-i y_{2}},
$$

is the complex field arising from the pair of wires alone (see Fig. 1) and $K_{z}(x)$ is the sheet current in the film. The complex potential describing the field generated by the wires alone, defined by $\mathcal{G}_{0}(\zeta)=\int_{0}^{\zeta} \mathcal{H}_{0}\left(\zeta^{\prime}\right) d \zeta^{\prime}$, is

$$
\mathcal{G}_{0}(\zeta)=\frac{I_{0}}{\pi} \ln \left(\frac{1+i \zeta / y_{1}}{1+i \zeta / y_{2}}\right)
$$

and the contour lines of the real part of $\mathcal{G}_{0}(\zeta)$ correspond to the magnetic field lines of $\mathcal{H}_{0}(\zeta)$. At the upper $(\zeta=x+i \epsilon)$ and lower $(\zeta=x-i \epsilon)$ surfaces of the superconducting film, (where we take $\epsilon=d / 2$ to be a positive infinitessimal, since $\left.d \ll y_{1},\right)$ the perpendicular and parallel magnetic fields $H_{y}(x, 0)=\operatorname{Re} \mathcal{H}(x \pm i \epsilon)$ and $H_{x}(x, \pm \epsilon)=\operatorname{Im} \mathcal{H}(x \pm i \epsilon)$ are obtained from Eq. (11) as

$$
\begin{aligned}
H_{y}(x, 0) & =H_{0 y}(x, 0)+\frac{\mathrm{P}}{2 \pi} \int_{-\infty}^{+\infty} d u \frac{K_{z}(u)}{x-u}, \\
H_{x}(x, \pm \epsilon) & =H_{0 x}(x, 0) \mp K_{z}(x) / 2,
\end{aligned}
$$

where $H_{0 y}(x, 0)=\operatorname{Re} \mathcal{H}_{0}(x), H_{0 x}(x, 0)=\operatorname{Im} \mathcal{H}_{0}(x)$, and $\mathrm{P}$ denotes the principal value integral. The complex potential is defined by $\mathcal{G}(\zeta)=\int_{i \epsilon}^{\zeta} \mathcal{H}\left(\zeta^{\prime}\right) d \zeta^{\prime}$, and the contour lines of the real part of $\mathcal{G}(\zeta)$ correspond to the magnetic field lines of $\mathcal{H}(\zeta)$.

\section{A. Meissner-state response}

We first consider the magnetic-field distribution when the film is in the (vortex-free) Meissner state and wires 1 and 2 carry dc currents $I_{1}=I_{0}>0$ and $I_{2}=-I_{0}<0$ after monotonically increasing in magnitude from zero. As discussed in Appendix A, when the current $I_{0}$ is small and the thickness $d$ is larger than $\lambda$, the magnetic field is practically zero below the film, where $y=\operatorname{Im} \zeta<0$. The field distribution above the film can be obtained by adding to $\mathcal{H}_{0}(\zeta)$ and $\mathcal{G}_{0}(\zeta)$ the contributions $\mathcal{H}_{I}(\zeta)$ and $\mathcal{G}_{I}(\zeta)$ due to image wires at $\zeta=-\zeta_{1}=-i y_{1}$ and $\zeta=$ $-\zeta_{2}=-i y_{2}$ carrying currents $-I_{0}$ and $+I_{0}$, respectively:

$$
\begin{aligned}
\mathcal{H}_{I}(\zeta) & =-\frac{I_{0}}{2 \pi} \frac{1}{\zeta+i y_{1}}+\frac{I_{0}}{2 \pi} \frac{1}{\zeta+i y_{2}}, \\
\mathcal{G}_{I}(\zeta) & =\frac{I_{0}}{\pi} \ln \left(\frac{1-i \zeta / y_{2}}{1-i \zeta / y_{1}}\right) .
\end{aligned}
$$

The resulting complex field $\mathcal{H}_{M}(\zeta)=\mathcal{H}_{0}(\zeta)+\mathcal{H}_{I}(\zeta)$ is

$$
\mathcal{H}_{M}(\zeta)= \begin{cases}i \frac{I_{0}}{\pi}\left(\frac{y_{1}}{\zeta^{2}+y_{1}^{2}}-\frac{y_{2}}{\zeta^{2}+y_{2}^{2}}\right) & \text { for } \operatorname{Im} \zeta>0, \\ 0 & \text { for } \operatorname{Im} \zeta<0 .\end{cases}
$$

The subscript $M$ is a reminder that this field describes the Meissner-state response to the applied field given in Eq. (2). Note that

$$
H_{M x}(x, \epsilon)=\frac{I_{0}}{\pi}\left(\frac{y_{1}}{x^{2}+y_{1}^{2}}-\frac{y_{2}}{x^{2}+y_{2}^{2}}\right) .
$$

As is usual using the method of images, $H_{M x}(x, \epsilon)=$ $2 H_{0 x}(x, 0)=2 \operatorname{Im} \mathcal{H}_{0}(x)$ [see Eq. (2)]. The corresponding complex potential $\mathcal{G}_{M}(\zeta)=\int_{i \epsilon}^{\zeta} \mathcal{H}_{M}\left(\zeta^{\prime}\right) d \zeta^{\prime}$ for $\operatorname{Im} \zeta>0$ is given by

$$
\mathcal{G}_{M}(\zeta)=i \frac{I_{0}}{\pi}\left[\arctan \left(\frac{\zeta}{y_{1}}\right)-\arctan \left(\frac{\zeta}{y_{2}}\right)\right] .
$$

We may take $\mathcal{G}_{M}(\zeta)=0$ for $\operatorname{Im} \zeta<0$. The perpendicular magnetic field and sheet-current density are thus given by $H_{M y}(x, 0)=0$ and

$$
K_{M z}(x)=-\frac{I_{0}}{\pi}\left(\frac{y_{1}}{x^{2}+y_{1}^{2}}-\frac{y_{2}}{x^{2}+y_{2}^{2}}\right) .
$$




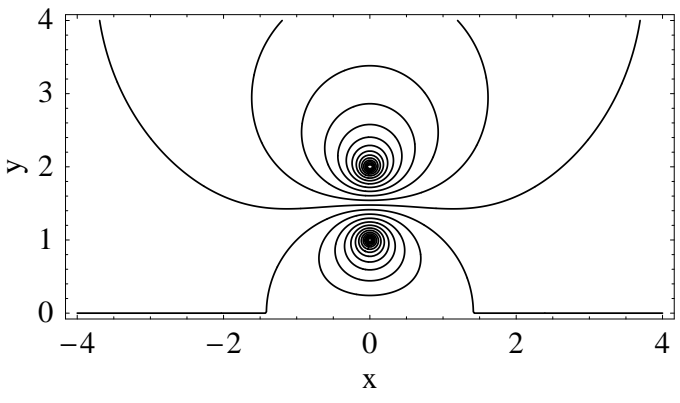

FIG. 2: Contour plot of the real part of the complex potential $\mathcal{G}_{M}(x+i y)$ vs $x$ and $y$ for $y_{1}=1$ and $y_{2}=2$. The contours correspond to magnetic field lines of the complex field $\mathcal{H}_{M}(x+i y)$ describing the Meissner-state response of the superconducting film to currents in the wires shown in Fig. 1. The contours near the wires at $\zeta=i y_{1}$ and $\zeta=i y_{2}$ are nearly circular.

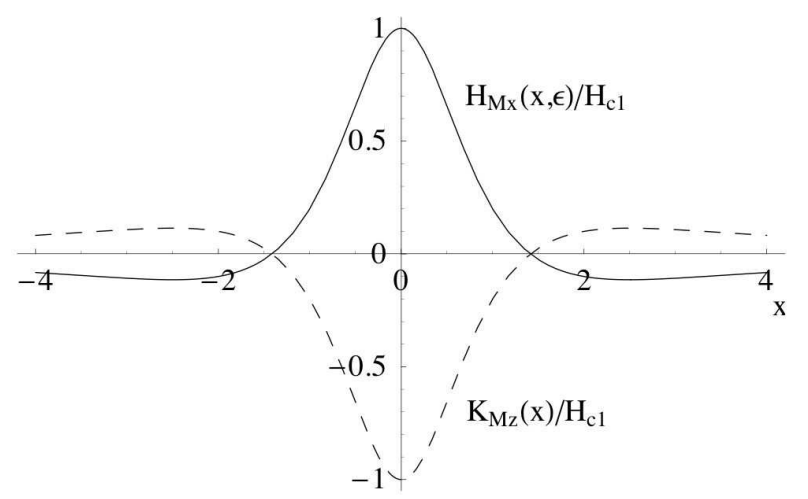

FIG. 3: Parallel magnetic field $H_{M x}(x, \epsilon) / H_{c 1}$ vs $x$ (solid curve) in the Meissner state at the top surface of the superconducting film when $I_{0}=I_{c 1}, H_{M x}(0, \epsilon)=H_{c 1}, y_{1}=1$, and $y_{2}=2$. The dashed curve shows the corresponding sheet-current density $K_{M z}(x) / H_{c 1}$ vs $x$. Note that $K_{M z}(0)=$ $-H_{c 1}$.

The net current induced in the superconducting film is $\int_{-\infty}^{+\infty} K_{M z}(x) d x=0$, as expected.

The maximum magnetic field parallel to the top surface of the film is

$$
H_{M x}(0, \epsilon)=\frac{I_{0}}{\pi}\left(\frac{1}{y_{1}}-\frac{1}{y_{2}}\right),
$$

and the maximum magnitude of the sheet-current density is

$$
\left|K_{M z}(0)\right|=\frac{I_{0}}{\pi}\left(\frac{1}{y_{1}}-\frac{1}{y_{2}}\right) .
$$

We now define two critical currents as follows. Let $I_{c 1}$ denote the value of $I_{0}$ at which $H_{M x}(0, \epsilon)$ in Eq. (12) reaches the lower critical field $H_{c 1}$,

$$
I_{c 1}=\frac{\pi H_{c 1} y_{1} y_{2}}{y_{2}-y_{1}},
$$

and let $I_{c 0}$ denote the value of $I_{0}$ at which $\left|K_{M z}(0)\right|$ in Eq. (13) reaches the critical sheet-current density $K_{c}$,

$$
I_{c 0}=\frac{\pi K_{c} y_{1} y_{2}}{y_{2}-y_{1}} .
$$

Both of these critical currents play important roles in determining the details of how magnetic flux penetrates through the film. In the following sections we discuss in turn the cases for which $K_{c}=0\left(I_{c 0}=0\right)$ and $K_{c}>0$ $\left(I_{c 0}>0\right)$.

\section{B. Vortex-generated fields and currents}

Consider a closely spaced row of vortices along the $z$ axis carrying magnetic flux $\Phi^{\prime}$ per unit length up through the film. Ignoring spatial variation on the scale of the London penetration depth $\lambda$ or the intervortex spacing, the magnetic field in the space $y>0(y<0)$ appears as if produced by a line of positive (negative) magnetic monopoles. At a distance $r$ from the origin, the magnitude of the magnetic field is $h=\Phi^{\prime} / \mu_{0} \pi r$. Expressing this result in terms of a complex magnetic field $\mathcal{H}_{v}(\zeta)=H_{v y}(x, y)+i H_{v x}(x, y)$ and extending it to a distribution of vortices or antivortices generating a magnetic field $H_{v y}(x, 0)$ in the plane of the film, we see that the vortex-generated complex magnetic field can be expressed as

$$
\mathcal{H}_{v}(\zeta)= \pm \frac{i}{\pi} \int_{-\infty}^{+\infty} d u \frac{H_{v y}(x, 0)}{\zeta-u}
$$

where $\zeta=x+i y$ and the upper (lower) sign in Eq. (16) holds for $y>0(y<0)$. The corresponding vortexgenerated sheet-current density $K_{v z}(x)=H_{v x}(x-i \epsilon)-$ $H_{v x}(x+i \epsilon)$ is

$$
K_{v z}(x)=-\frac{\mathrm{P}}{\pi} \int_{-\infty}^{+\infty} d u \frac{2 H_{v y}(x, 0)}{x-u}
$$

while the Biot-Savart law yields another relation between $K_{v z}(x)$ and $H_{v y}(x, 0)$ :

$$
2 H_{v y}(x, 0)=\frac{\mathrm{P}}{\pi} \int_{-\infty}^{+\infty} d u \frac{K_{v z}(u)}{x-u} .
$$

In the following sections, the complex field can always be regarded as a linear superposition of the Meissner-state and vortex-generated complex fields: $\mathcal{H}(\zeta)=\mathcal{H}_{M}(\zeta)+\mathcal{H}_{v}(\zeta)$. The complex potential $\mathcal{G}(\zeta)=$ $\int_{i \epsilon}^{\zeta} \mathcal{H}\left(\zeta^{\prime}\right) d \zeta^{\prime}$ can be written as $\mathcal{G}(\zeta)=\mathcal{G}_{M}(\zeta)+\mathcal{G}_{v}(\zeta)$. Similarly, the sheet-current density can always be expressed as $K_{z}(x)=K_{M z}(x)+K_{v z}(x)$.

\section{Flux domes in the absence of bulk pinning}

In bulk-pinning-free films $\left(K_{c}=0\right)$, the first vortex enters the film at $x=0$, where the maximum magnetic field 
at the top surface is equal to the lower critical field $H_{c 1}{ }^{22}$ This occurs at the current $I_{0}=I_{c 1}$, given in Eq. (14) (see Fig. 3). An initially tiny vortex loop expands in radius, and a portion of the loop is driven to the bottom of the film surface, where it annihilates, resulting in a separated vortex-antivortex pair. The vortex (antivortex) carries magnetic flux $\phi_{0}$ in the $+y(-y)$ direction, where $\phi_{0}=h / 2 e$ is the superconducting flux quantum. Responding to the Lorentz force $K_{M z}(x) \phi_{0}$, the vortex moves in the $x$ direction until it comes to rest at the point $x=x_{0}=\sqrt{y_{1} y_{2}}$, where $K_{M z}(x)=0$, as can be seen from Eq. (11) and Fig. 3. The antivortex moves in the opposite direction and comes to rest at the point $x=-x_{0}$.

For increasing values of $I_{0}$ in the range $I_{c 1}<I_{0}<$ $2 I_{c 1}$, the magnetic field distribution perpendicular to the film can be characterized as having a positive vortexgenerated magnetic flux dome in the region $b<x<a$, where $0<b<x_{0}<a$, and a negative antivortexgenerated flux dome in the region $-a<x<-b$. The complex magnetic field $\mathcal{H}(\zeta)=\mathcal{H}_{M}(\zeta)+\mathcal{H}_{v}(\zeta)$ is given in Eq. (B13) but with $K_{c}=0$. Subtracting the Meissnerstate complex field $\mathcal{H}_{M}(\zeta)$, we obtain the following expression for the vortex-generated complex magnetic field,

$$
\mathcal{H}_{v}(\zeta)=\frac{I_{0}}{2 \pi}\left\{\frac{y_{1}\left[\mp i+\phi(\zeta) / s_{1}\right]}{\zeta^{2}+y_{1}^{2}}-\frac{y_{2}\left[\mp i+\phi(\zeta) / s_{2}\right]}{\zeta^{2}+y_{2}^{2}}\right\}
$$

where $s_{j}=\sqrt{\left(a^{2}+y_{j}^{2}\right)\left(b^{2}+y_{j}^{2}\right)}$, and the upper (lower) sign holds when $\zeta=x+i y$ is in the upper (lower) half plane. Writing $\phi(\zeta)=\phi_{1}(x, y)+i \phi_{2}(x, y)$, we find that $\phi_{1}(x, y)$ is an odd function of $x$ and an even function of $y\left[\phi_{1}(x, y)=-\phi_{1}(-x, y)=\phi_{1}(x,-y)\right]$, while $\phi_{2}(x, y)$ is an even function of $x$ and an odd function of $y\left[\phi_{2}(x, y)=\phi_{2}(-x, y)=-\phi_{2}(x,-y)\right]$. Thus $H_{v y}(x, y)$ is an odd function of $x$ but an even function of $y$, while $H_{v x}(x, y)$ is an odd function of $y$ but an even function of $x$. Just above (below) the real axis,

$$
\phi(x \pm i \epsilon)= \begin{cases} \pm i \tilde{\phi}(x), & |x|<b \text { or }|x|>a, \\ \tilde{\phi}(x), & b<|x|<a,\end{cases}
$$

where

$$
\tilde{\phi}(x)= \begin{cases}\sqrt{\left(a^{2}-x^{2}\right)\left(b^{2}-x^{2}\right)}, & |x|<b, \\ \operatorname{sgn}(x) \sqrt{\left(a^{2}-x^{2}\right)\left(x^{2}-b^{2}\right)}, & b<|x|<a, \\ -\sqrt{\left(x^{2}-a^{2}\right)\left(x^{2}-b^{2}\right)}, & |x|>a .\end{cases}
$$

From Eq. (19) we obtain the following values of $H_{v y}(x, 0)$, $H_{v x}(x, \epsilon)=-H_{v x}(x,-\epsilon)$, and $K_{v z}(x)=H_{v x}(x,-\epsilon)-$ $H_{v x}(x, \epsilon)$,

$$
H_{v y}(x, 0)= \begin{cases}0, & |x|<b \text { or }|x|>a, \\ \frac{I_{0}}{2 \pi}\left(\frac{y_{1}}{s_{1}\left(x^{2}+y_{1}^{2}\right)}\right. & \\ \left.-\frac{y_{2}}{s_{2}\left(x^{2}+y_{2}^{2}\right)}\right) \tilde{\phi}(x), & b<|x|<a,\end{cases}
$$

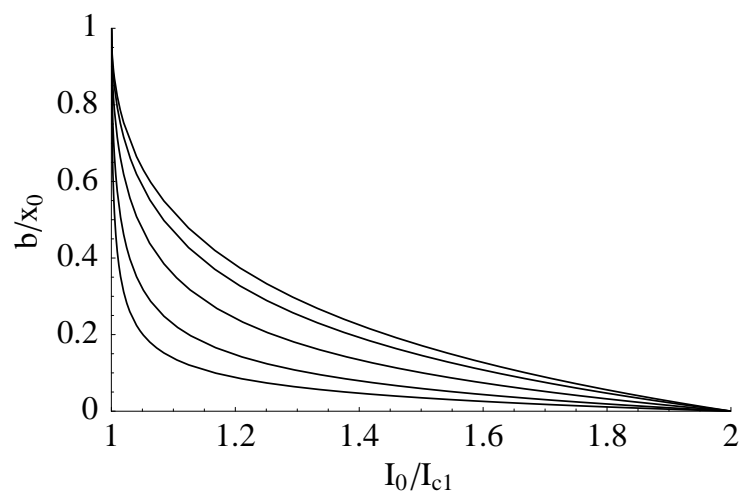

FIG. 4: Plot of the vortex dome's left boundary $b$ vs $I_{0} / I_{c 1}$ for the bulk-pinning-free case, where $b$ is in units of $x_{0}=\sqrt{y_{1} y_{2}}$, for $I_{c 1}<I_{0}<2 I_{c 1}$ and $y_{1} / y_{2}=0.01,0.03,0.1,0.3$, and 1 (bottom to top). The right boundary of the vortex dome is at $a=x_{0}^{2} / b$.

$$
\begin{aligned}
& H_{v x}(x, \epsilon)=\left\{\begin{array}{c}
-\frac{I_{0}}{2 \pi}\left(\frac{y_{1}\left[1-\tilde{\phi}(x) / s_{1}\right]}{x^{2}+y_{1}^{2}}-\frac{y_{2}\left[1-\tilde{\phi}(x) / s_{2}\right]}{x^{2}+y_{2}^{2}}\right), \\
|x|<b \text { or }|x|>a, \\
-\frac{I_{0}}{2 \pi}\left(\frac{y_{1}}{x^{2}+y_{1}^{2}}-\frac{y_{2}}{x^{2}+y_{2}^{2}}\right), b<|x|<a,
\end{array}\right. \\
& K_{v z}(x)=\left\{\begin{array}{l}
\frac{I_{0}}{\pi}\left(\frac{y_{1}\left[1-\tilde{\phi}(x) / s_{1}\right]}{x^{2}+y_{1}^{2}}-\frac{y_{2}\left[1-\tilde{\phi}(x) / s_{2}\right]}{x^{2}+y_{2}^{2}}\right), \\
|x|<b \text { or }|x|>a, \\
\frac{I_{0}}{\pi}\left(\frac{y_{1}}{x^{2}+y_{1}^{2}}-\frac{y_{2}}{x^{2}+y_{2}^{2}}\right), \quad b<|x|<a,
\end{array}\right.
\end{aligned}
$$

As discussed in Appendix $\mathrm{B}$, the requirement that $\int_{-\infty}^{+\infty} K_{z}(x) d x=0$ leads to the condition that $a b=x_{0}^{2}=$ $y_{1} y_{2}$ when there is no bulk pinning. A second condition relating $a, b$ and $I_{0}$ follows from the requirement that $H_{x}(0, \epsilon)=H_{M x}(0, \epsilon)+H_{v x}(0, \epsilon)=H_{c 1}$, which yields

$$
I_{0}=\left(\frac{2}{1+y_{1} y_{2}\left(s_{1}+s_{2}\right) / s_{1} s_{2}}\right) I_{c 1} .
$$

Combining these two conditions and eliminating $a$, we obtain the following connection between $I_{0}$ and $b$,

$$
I_{0}=\left(\frac{2}{1+b\left(y_{1}+y_{2}\right) / \sqrt{\left(y_{1}^{2}+b^{2}\right)\left(y_{2}^{2}+b^{2}\right)}}\right) I_{c 1} .
$$

When $I_{0}=I_{c 1}, a=b=x_{0}=\sqrt{y_{1} y_{2}}$. As $I_{0}$ increases above $I_{c 1}, b$ decreases monotonically, as shown in Fig. 目 and the lower solid curve in Fig. 11] In the limit as $I_{0} \rightarrow 2 I_{c 1}$, we see that $b \rightarrow 0$, such that $a=y_{1} y_{2} / b \rightarrow \infty$. For $I_{0}>2 I_{c 1}$, the magnetic-flux-filled region extends from $-\infty$ to $+\infty, K_{z}(x)=0$, and $\mathcal{H}(\zeta)=\mathcal{H}_{0}(\zeta)$ everywhere, since the superconducting film is then completely incapable of screening the magnetic field produced by the two wires.

Figures 5 and 6 show plots of $H_{y}(x, 0)=H_{v y}(x, 0)$ and $K_{z}(x)$ vs $x$ for several values of $I_{0}$ in the range $I_{c 1} \leq I_{0} \leq$ $2 I_{c 1}$. 


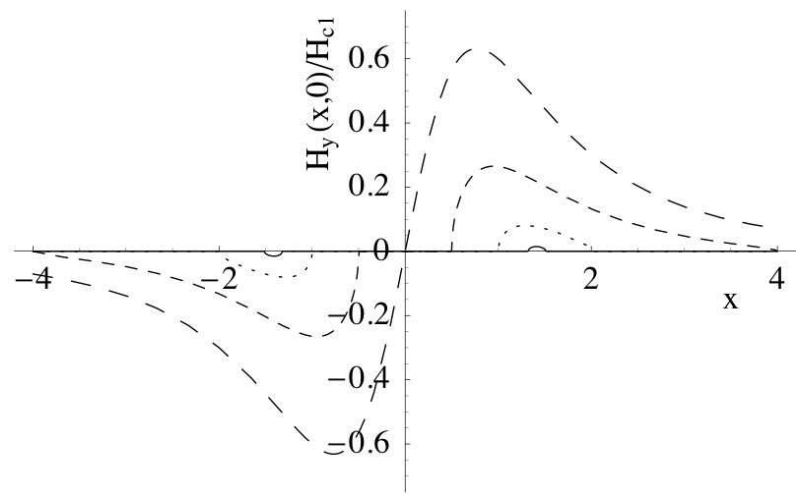

FIG. 5: The perpendicular magnetic field $H_{y}(x, 0) / H_{c 1}=$ $H_{v y}(x, 0) / H_{c 1}$ vs $x$ exhibits vortex and antivortex flux domes at $b<|x|<a$ in the absence of bulk pinning. With $y_{1}=$ $1, y_{2}=2$, and $x_{0}=\sqrt{y_{1} y_{2}}=1.414$, when $I_{0}=1.001 I_{c 1}$, tiny domes are centered at $\pm x_{0}$. As $I_{0}$ increases, the domes become taller and wider, as shown for $I_{0}=1.026 I_{c 1}$ when $b=1$ (dotted), $I_{0}=1.212 I_{c 1}$ when $b=0.5$ (short dash), and $I_{0}=2 I_{c 1}$ when $b=0$ and $a=\infty$ (long dash). For $I_{0} \geq 2 I_{c 1}$, the superconducting film is no longer capable of screening, $K_{z}(x)=0$ everywhere, and $H_{y}(x, 0)=H_{0 y}(x, 0)$ [Eq. (2)].

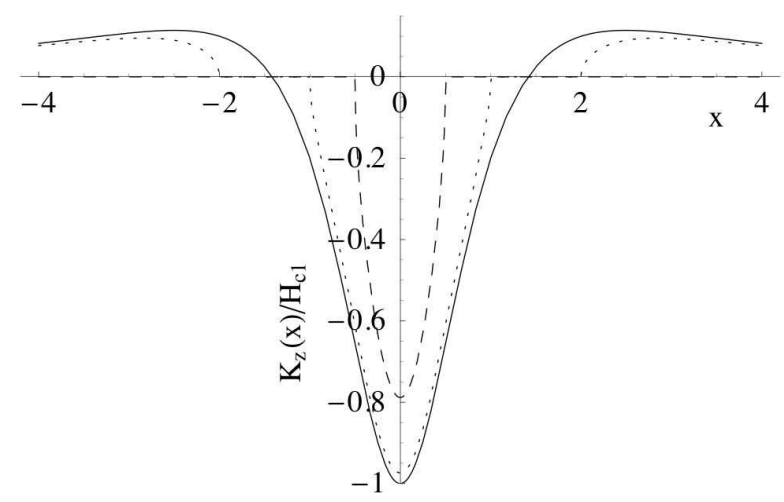

FIG. 6: Sheet-current-density $K_{z}(x) / H_{c 1}$ vs $x$ in the absence of bulk pinning for $y_{1}=1, y_{2}=2$, and $x_{0}=\sqrt{y_{1} y_{2}}=1.414$. When $I_{0}=I_{c 1}, K_{z}(x)$ (solid) is equal to $K_{M z}(x)$, shown in Fig. 3. With increasing $I_{0}$, regions of $K_{z}(x)=0$ develop underneath the vortex and antivortex domes at $b<|x|<a$, beginning at $x= \pm x_{0}$. Shown are results for $I_{0}=1.026 I_{c 1}$ when $b=1$ (dotted) and $I_{0}=1.212 I_{c 1}$ when $b=0.5$ (dashed). For $I_{0} \geq 2 I_{c 1}$, the superconducting film is no longer capable of screening, and $K_{z}(x)=0$ everywhere.

Figure 7 shows plots of $H_{x}(0, \pm \epsilon)=H_{M x}(0, \pm \epsilon)+$ $H_{v x}(0, \pm \epsilon)$ vs $I_{0}$. For $0 \leq I_{0} \leq I_{c 1}$, we have $H_{x}(0, \epsilon)=H_{M x}(0, \epsilon)=H_{c 1} I_{0} / I_{c 1}$ and $H_{x}(0,-\epsilon)=$ $H_{M x}(0,-\epsilon)=0$. For $I_{c 1} \leq I_{0} \leq 2 I_{c 1}$, we have $H_{x}(0, \epsilon)=H_{M x}(0, \epsilon)+H_{v x}(0, \epsilon)=H_{c 1}$ and $H_{x}(0,-\epsilon)=$ $H_{v x}(0,-\epsilon)=H_{c 1}\left(I_{0} / I_{c 1}-1\right)$, where $H_{M x}(0, \epsilon)=$ $H_{c 1} I_{0} / I_{c 1}$ and $H_{v x}(0, \pm \epsilon)= \pm H_{c 1}\left(1-I_{0} / I_{c 1}\right)$. When $I_{0}=2 I_{c 1}, H_{x}(0, \pm \epsilon)=H_{c 1}$. For $I_{0}>I_{c 1}, H_{x}(0, \pm \epsilon)=$ $H_{0 x}(0,0)=H_{c 1} I_{0} / 2 I_{c 1}$, and in addition $H_{x}(x, \pm \epsilon)=$ $H_{M x}(x, \pm \epsilon)+H_{v x}(x, \pm \epsilon)=H_{0 x}(x, 0)$ for all $x$. The macroscopic magnetic-field distribution is then the same

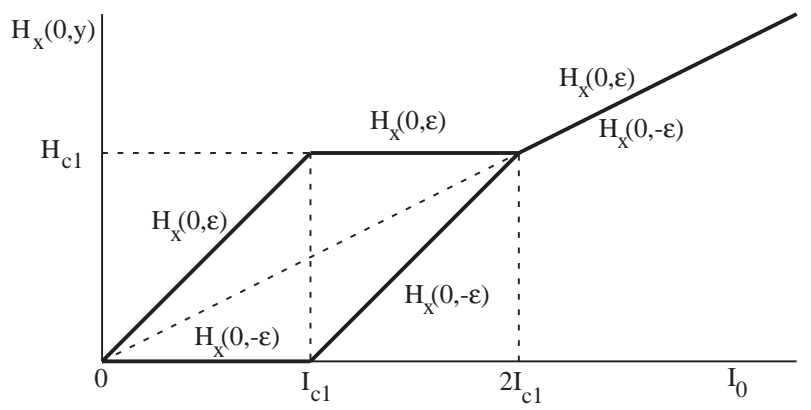

FIG. 7: Parallel magnetic fields at $x=0$ at the top and bottom surfaces $H_{x}(0, \epsilon)$ and $H_{x}(0,-\epsilon)$ vs $I_{0}$ in the absence of bulk pinning. When $0 \leq I_{0} \leq I_{c 1}, H_{x}(0, \epsilon)=H_{M x}(0, \epsilon)$ [Eq. (8)] and $H_{x}(0,-\epsilon)=H_{M x}(0,-\epsilon)=0$. When $I_{c 1} \leq$ $I_{0} \leq 2 I_{c 1}, H_{x}(0, \epsilon)=H_{M x}(0, \epsilon)+H_{v x}(0, \epsilon)=H_{c 1}$, and $H_{x}(0,-\epsilon)=H_{v x}(0,-\epsilon)=-H_{v x}(0, \epsilon)$. When $I_{0} \geq 2 I_{c 1}$, $H_{x}(0, \pm \epsilon)=H_{0 x}(0,0)=H_{c 1} I_{0} / 2 I_{c 1}$ [Eq. (2)].

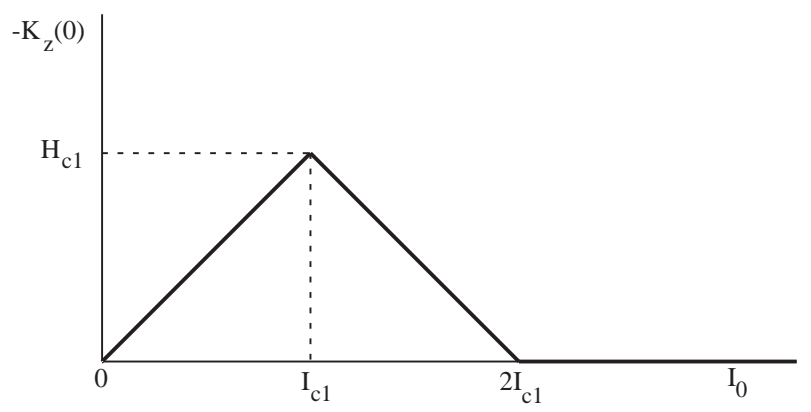

FIG. 8: The magnitude of the sheet-current-density at the origin $-K_{z}(0)=H_{x}(0, \epsilon)-H_{x}(0,-\epsilon)$ vs $I_{0}$ in the absence of bulk pinning (see Fig. 7).

as it would be if the superconducting film were absent.

Figure 8 shows a plot of $-K_{z}(0)$ vs $I_{0}$, where $K_{z}(0)=$ $H_{x}(0,-\epsilon)-H_{x}(0, \epsilon)$. For $0 \leq I_{0} \leq I_{c 1},-K_{z}(0)=$ $H_{c 1} I_{0} / I_{c 1}$, and for $I_{c 1} \leq I_{0} \leq 2 I_{c 1},-K_{z}(0)=H_{c 1}(2-$ $\left.I_{0} / I_{c 1}\right)$. For $I_{0}>2 I_{c 1}, K_{z}(x)=0$ for all $x$, i.e., everywhere in the film.

The vortex-generated complex potential $\mathcal{G}_{v}(\zeta)=$ $\mathcal{G}(\zeta)-\mathcal{G}_{M}(\zeta)$ [see Eqs. (10) and (B14)] in the absence of bulk pinning is

$$
\mathcal{G}_{v}(\zeta)=\frac{I_{0}}{2 \pi}\left[g_{v}\left(\zeta, y_{1}\right)-g_{v}\left(\zeta, y_{2}\right)\right]
$$

where

$$
\begin{aligned}
g_{v}(\zeta, y)= & \mp i \arctan (\zeta / y) \\
& \mp \frac{i}{a s y}\left[a^{2} y^{2} \boldsymbol{E}(\theta, k)+y^{2}\left(b^{2}+y^{2}\right) \boldsymbol{F}(\theta, k)\right. \\
& \left.-\left(a^{2}+y^{2}\right)\left(b^{2}+y^{2}\right) \Pi\left(\theta,-b^{2} / y^{2}, k\right)\right], \\
s= & \sqrt{\left(a^{2}+y^{2}\right)\left(b^{2}+y^{2}\right)} \\
\theta= & \arcsin (\zeta / b), \text { and } \\
k= & b / a
\end{aligned}
$$

where $\boldsymbol{E}, \boldsymbol{K}$, and $\boldsymbol{\Pi}$ are incomplete elliptic integrals and the upper (lower) signs hold in the upper (lower) half $\zeta$ 


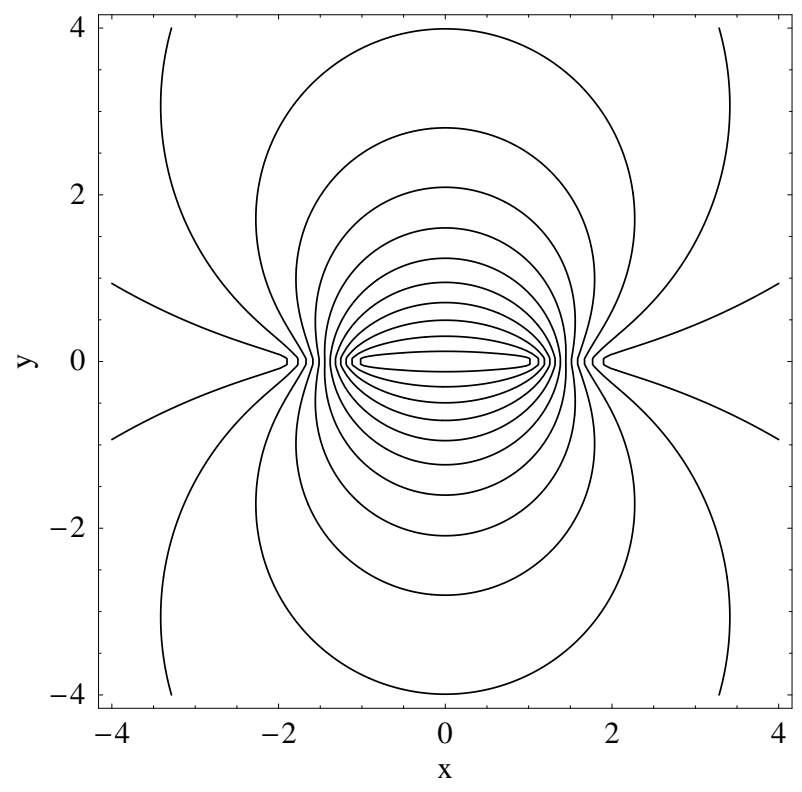

FIG. 9: Contour plot of the real part of the vortex-generated complex potential in the absence of bulk pinning [Eq. (27)] $\mathcal{G}_{v}(x+i y)$ vs $x$ and $y$ for $y_{1}=1, y_{2}=2, a=2$, and $b=1$, as for the dotted curves in Figs. 5 and 6 . The contours correspond to magnetic field lines of the vortex-generated complex field $\mathcal{H}_{v}(x+i y)$ given in Eq. (19).

plane. Shown in Fig. 9 is a contour plot of the real part of $\mathcal{G}_{v}(x+i y)$. These contours correspond to the magnetic field lines of the vortex-generated magnetic field. The magnetic field flows in a generally counterclockwise direction, carried by vortices in the region $b<x<a$ up through the film and by antivortices in the region $-a<x<-b$ back down through the film. A contour plot of the real part of $\mathcal{G}(x+i y)=\mathcal{G}_{M}(x+i y)+\mathcal{G}_{v}(x+i y)$ would also show the magnetic field lines generated by the two wires, as in Fig. 2.

\section{Flux domes in the presence of weak bulk pinning}

In superconducting films in which bulk pinning is present and is characterized by a field-independent critical sheet-current density $K_{c}=j_{c} d>0$, the first vortex enters the film at $x=0$, when the maximum parallel magnetic field at the top surface $H_{M x}(0, \epsilon)$ [Eq. [12] ] is equal to the lower critical field $H_{c 1} \stackrel{22}{2}$ As in Sec. [II] this again occurs at the current $I_{0}=I_{c 1}$, given in Eq. (14). According to critical-state theory, 23,24 this vortex advances toward the bottom surface at the leading edge of a curving flux front of nearly parallel vortices of thickness $d_{p}=\left[H_{M x}(0, \epsilon)-H_{c 1}\right] / j_{c}$, and it reaches the bottom surface when $d_{p}=d$, i.e., when $H_{M x}(0, \epsilon)=H_{c 1}+K_{c}$ or $I_{0}=I_{c 1}+I_{c 0}$ [see Eqs. (12), (14), and (15)]. The positive end of the first vortex is then at $x=x_{p}$ and its negative end is at $x=-x_{p}$, where $x_{p}$ is the solution of
$H_{M x}\left(x_{p}, \epsilon\right)=H_{c 1}$ [see Eq. (92)]. It can be shown that $x_{p} \gg d$ when $d \ll y_{1}$ except when $K_{c} \ll H_{c 1}$.

Once the first vortex reaches the bottom surface, the portion at $x \approx 0$ annihilates with its image, and the vortex divides into two halves. The half in the region $x>0$, which we call a vortex, carries magnetic flux $\phi_{0}$ $u p$ from the bottom to the top surface, and the half in the region $x<0$, which we call an antivortex, carries magnetic flux $\phi_{0}$ down from the top to the bottom surface. Since $H_{M x}(0,-\epsilon)=0$ and $H_{M x}(0, \epsilon)=H_{c 1}+K_{c}$ at $I_{0}=I_{c 1}+I_{c 0}$, the sheet-current density is initially $K_{M z}(0)=-H_{c 1}-K_{c}$. Because $\left|K_{M z}(0)\right|>K_{c}$, the vortex separates from the rest of the flux front and is driven in the $x$ direction by the Lorentz force $K_{M z}(x) \phi_{0}$ until it comes to rest at $x=x_{c}$, where $\left|K_{M z}\left(x_{c}\right)\right|=K_{c}$ [see Eq. (11)] and the Lorentz force is balanced by the pinning force. Similarly, the antivortex moves in the opposite direction and comes to rest at the point $x=-x_{c}$. As we will show below, $0<x_{c}<x_{0}=\sqrt{y_{1} y_{2}}$ when $K_{c}>0$ [see Fig. 10].

For increasing values of $I_{0}$ in the range $I_{c 1}+I_{c 0}<I_{0}<$ $2 I_{c 1}+I_{c 0}$, the magnetic field distribution perpendicular to the film can be characterized as having a positive vortexgenerated magnetic flux dome in the region $b<x<$ $a$, where $0<b<x_{c}<a$, and a negative antivortexgenerated flux dome in the region $-a<x<-b$. The complex magnetic field $\mathcal{H}(\zeta)=\mathcal{H}_{M}(\zeta)+\mathcal{H}_{v}(\zeta)$ is given by Eq. (B13). Subtracting the Meissner-state complex field $\mathcal{H}_{M}(\zeta)$, we obtain the following expression for the vortex-generated complex magnetic field,

$$
\begin{aligned}
\mathcal{H}_{v}(\zeta)= & \frac{I_{0}}{2 \pi}\left\{\frac{y_{1}\left[\mp i+\phi(\zeta) / s_{1}\right]}{\zeta^{2}+y_{1}^{2}}-\frac{y_{2}\left[\mp i+\phi(\zeta) / s_{2}\right]}{\zeta^{2}+y_{2}^{2}}\right\} \\
& \pm i K_{c} / 2
\end{aligned}
$$

where $s_{j}=\sqrt{\left(a^{2}+y_{j}^{2}\right)\left(b^{2}+y_{j}^{2}\right)}$, and the upper (lower) sign holds when $\zeta=x+i y$ is in the upper (lower) half plane. Following a procedure similar to that used in Sec. IIC we obtain the following values of $H_{v y}(x, 0)$, $H_{v x}(x, \epsilon)=-H_{v x}(x,-\epsilon)$, and $K_{v z}(x)=H_{v x}(x,-\epsilon)-$ $H_{v x}(x, \epsilon)$,

$$
H_{v y}(x, 0)= \begin{cases}0, & |x|<b \text { or }|x|>a \\ \frac{I_{0}}{2 \pi}\left(\frac{y_{1}}{s_{1}\left(x^{2}+y_{1}^{2}\right)}\right. & \\ \left.-\frac{y_{2}}{s_{2}\left(x^{2}+y_{2}^{2}\right)}\right) \tilde{\phi}(x), & b<|x|<a\end{cases}
$$

$H_{v x}(x, \epsilon)=\left\{\begin{array}{c}-\frac{I_{0}}{2 \pi}\left(\frac{y_{1}\left[1-\tilde{\phi}(x) / s_{1}\right]}{x^{2}+y_{1}^{2}}-\frac{y_{2}\left[1-\tilde{\phi}(x) / s_{2}\right]}{x^{2}+y_{2}^{2}}\right) \\ +K_{c} / 2, \quad|x|<b \text { or }|x|>a, \\ -\frac{I_{0}}{2 \pi}\left(\frac{y_{1}}{x^{2}+y_{1}^{2}}-\frac{y_{2}}{x^{2}+y_{2}^{2}}\right)+K_{c} / 2, \\ b<|x|<a,\end{array}\right.$ 


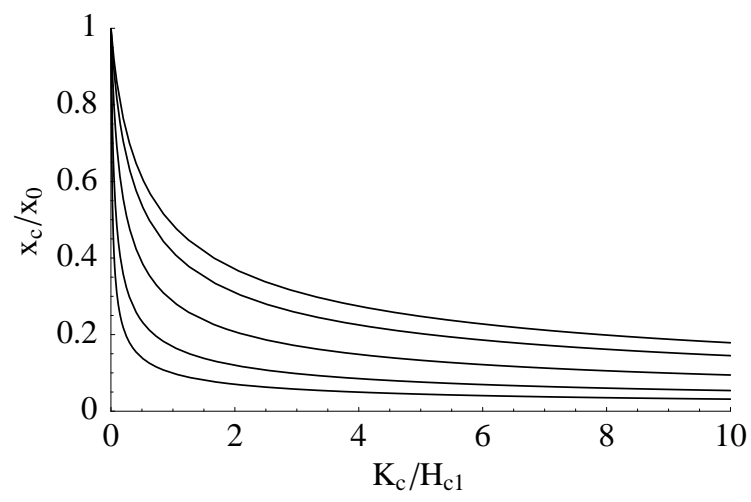

FIG. 10: Plot of $\tilde{x}_{c}=x_{c} / x_{0}$ vs $K_{c} / H_{c 1}$ in the presence of bulk pinning, where $x_{c}$ is the position of the first entering vortex when $I_{0}$ just exceeds $I_{c 1}+I_{c 0}$ and $x_{0}=\sqrt{y_{1} y_{2}}$. Curves are shown for $y_{1} / y_{2}=0.01,0.03,0.1,0.3$, and 1 (bottom to top).

$K_{v z}(x)=\left\{\begin{array}{l}\frac{I_{0}}{\pi}\left(\frac{y_{1}\left[1-\tilde{\phi}(x) / s_{1}\right]}{x^{2}+y_{1}^{2}}-\frac{y_{2}\left[1-\tilde{\phi}(x) / s_{2}\right]}{x^{2}+y_{2}^{2}}\right) \\ -K_{c}, \quad|x|<b \text { or }|x|>a, \\ \frac{I_{0}}{\pi}\left(\frac{y_{1}}{x^{2}+y_{1}^{2}}-\frac{y_{2}}{x^{2}+y_{2}^{2}}\right)-K_{c}, b<|x|<a,\end{array}\right.$

For $I_{c 1}+I_{c 0}<I_{0}<2 I_{c 1}+I_{c 0}$, the requirement that $\int_{-\infty}^{+\infty} K_{z}(x) d x=0$ leads to Eq. B24). A second condition on $a, b$, and $I_{0}$ follows from the requirement that $H_{x}(0, \epsilon)=H_{c 1}+K_{c}$, which from Eq. (B13) yields:

$$
\begin{aligned}
\mathcal{H}_{x}(0, \epsilon) & =\frac{I_{0}}{2 \pi}\left[\frac{\left(1+a b / s_{1}\right)}{y_{1}}-\frac{\left(1+a b / s_{2}\right)}{y_{2}}\right]+\frac{K_{c}}{2} \\
& =H_{c 1}+K_{c} .
\end{aligned}
$$

Elimination of $I_{0}$ between Eqs. (B24) and (36) yields the equation

$$
\frac{y_{1} y_{2}\left(y_{1} s_{2}-y_{2} s_{1}\right)}{\left(y_{2}-y_{1}\right) s_{1} s_{2}+a b\left(y_{2} s_{2}-y_{1} s_{1}\right)}=\frac{K_{c} / 2 H_{c 1}}{1+K_{c} / 2 H_{c 1}} .
$$

Numerical solutions of Eqs. (36) and (37) yield $a$ and $b$ as a function of $I_{0}$ for any given value of $K_{c}$. As discussed above, when $I_{0}$ just exceeds $I_{c 1}+I_{c 0}$, the first vortex (antivortex) comes to rest at $x_{c}\left(-x_{c}\right)$. The equation determining the value of $\tilde{x}_{c}=x_{c} / x_{0}=x_{c} / \sqrt{y_{1} y_{2}}$ can be obtained from Eq. (B24) by setting $a=b=x_{c}$ and making use of Eqs. (144) and (15):

$$
\frac{\left(1-\tilde{x}_{c}^{2}\right)}{\left(\tilde{x}_{c}^{2}+y_{1} / y_{2}\right)\left(\tilde{x}_{c}^{2}+y_{2} / y_{1}\right)}=\frac{K_{c} / H_{c 1}}{1+K_{c} / H_{c 1}} .
$$

Figure 10 shows plots of $\tilde{x}_{c}$ vs $K_{c} / H_{c 1}$ for various values of $y_{1} / y_{2}$, obtained by numerically solving Eq. (38). Note that for each case $\tilde{x}_{c}=1$ when $K_{c}=0$, and $\tilde{x}_{c} \rightarrow 0$ when $K_{c} / H_{c 1} \rightarrow \infty$. For $K_{c} / H_{c 1} \gg 1$,

$$
\tilde{x}_{c} \approx 1 / \sqrt{\left(1+y_{1} / y_{2}+y_{2} / y_{1}\right)\left(K_{c} / H_{c 1}\right)} .
$$

Numerical solutions for $a$ and $b$ when $I_{c 1}+I_{c 0}<I_{0}<$ $2 I_{c 1}+I_{c 0}$ are shown by the dotted curves in Fig. 11 for

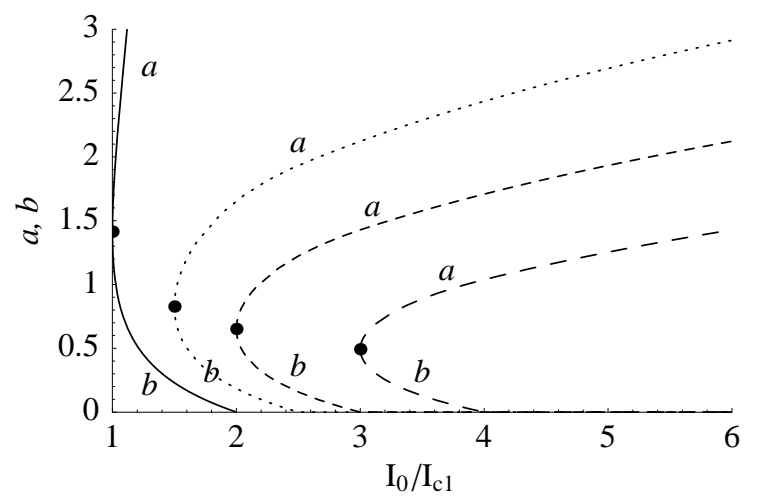

FIG. 11: Plots of $a$ (upper curves), the right boundary of the vortex dome, and $b$ (lower curves), the left boundary, vs $I_{0} / I_{c 1}$ for $y_{1}=1$ and $y_{2}=2: K_{c}=0$ (solid curves separated by the dot at $\left.a=b=x_{c}=1.414\right), K_{c}=H_{c 1} / 2$ (dotted curves and dot at $\left.x_{c}=0.827\right), K_{c}=H_{c 1}$ (short-dashed curves and dot at $x_{c}=0.651$ ), and $K_{c}=2 H_{c 1}$ (long-dashed curves and dot at $\left.x_{c}=0.493\right)$. Note that $b=0$ when $I_{0} / I_{c 1} \geq 2+K_{c} / H_{c 1}$.

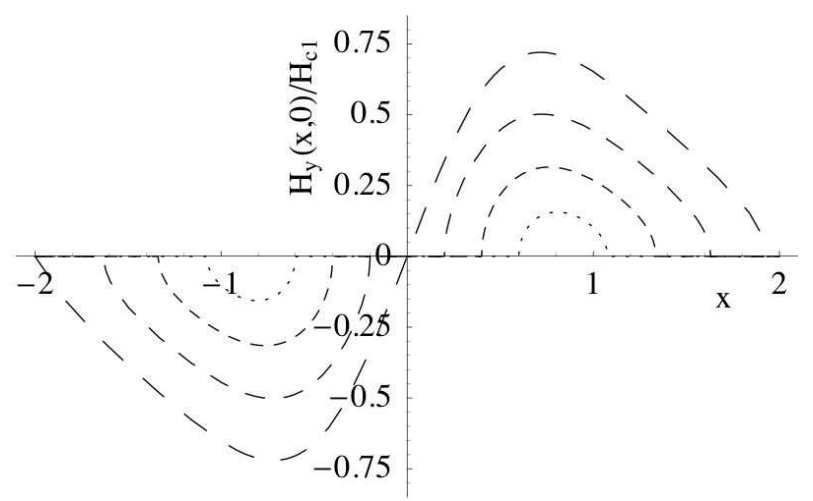

FIG. 12: When $I_{0}>I_{c 1}+I_{c 0}$, the perpendicular magnetic field $H_{y}(x, 0) / H_{c 1}=H_{v y}(x, 0) / H_{c 1}$ vs $x$ initially exhibits separated vortex and antivortex flux domes at $b<|x|<a$ even in the presence of bulk pinning, as shown here for $K_{c}=H_{c 1} / 2$, $y_{1}=1, y_{2}=2$, and $x_{c}=0.827$. When $I_{0}$ is just above $I_{c 1}+I_{c 0}=1.5 I_{c 1}$, tiny domes are centered at $\pm x_{c}$. As $I_{0}$ increases, the domes become taller and wider, as shown for $I_{0}=1.545 I_{c 1}$ when $b=0.600$ and $a=1.077$ (dotted curves), $I_{0}=1.683 I_{c 1}$ when $b=0.400$ and $a=1.337$ (shortdashed curves), $I_{0}=1.966 I_{c 1}$ when $b=0.200$ and $a=1.630$ (medium-dashed curves), and $I_{0}=2 I_{c 1}+I_{c 0}=2.5 I_{c 1}$ when $b=0$ and $a=1.933$ (long-dashed curves).

the case that $y_{1}=1, y_{2}=2, x_{0}=\sqrt{2}$, and $K_{c}=H_{c 1} / 2$, such that $I_{c 0}=I_{c 1} / 2$. Note that $a=b=x_{c}=0.585 x_{0}=$ 0.827 at $I_{0}=I_{c 1}+I_{c 0}$ when vortex penetration first occurs. From Eqs. (36), (14), and (15), we see that $b=0$ when $I_{0}=2 I_{c 1}+I_{c 0}$. When $I_{0}>2 I_{c 1}+I_{c 0}, b$ remains equal to zero, and the value of $a$ must be obtained from Eq. B25. Figure 11 also shows similar plots of $a$ and $b$ for $K_{c}=H_{c 1}$ and $K_{c}=2 H_{c 1}$

Figures 12 and 13 show plots of $H_{y}(x, 0)=H_{v y}(x, 0)$ and $K_{z}(x)$ vs $x$ for several values of $I_{0}$ in the range $I_{c 1}+$ $I_{c 0}<I_{0} \leq 2 I_{c 1}+I_{c 0}$. 


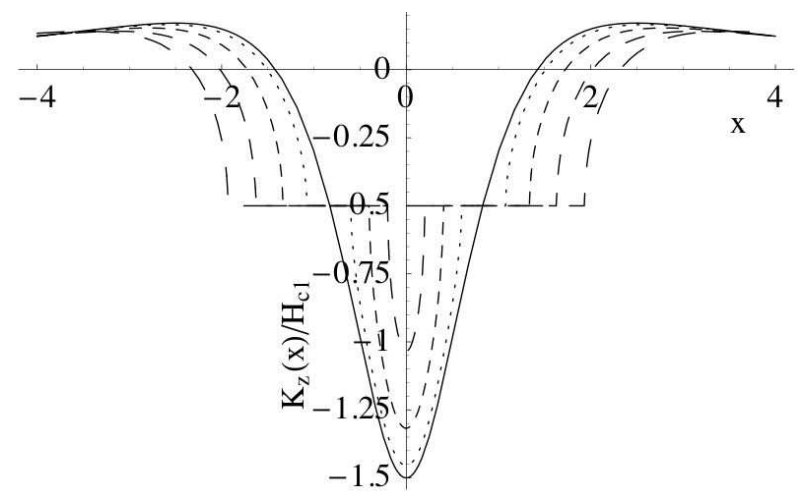

FIG. 13: Sheet-current density $K_{z}(x, 0) / H_{c 1}$ vs $x$ in the presence of bulk pinning, shown here for $K_{c}=H_{c 1} / 2, y_{1}=1$, $y_{2}=2$, and $x_{c}=0.827$. When $I_{0}=I_{c 1}+I_{c 0}, K_{z}(x)$ (solid) is equal to $K_{M z}(x)$, shown in Fig. 3 . With increasing $I_{0}$, regions of $K_{z}(x)=-K_{c}$ develop underneath the vortex and antivortex domes at $b<|x|<a$, beginning at $x= \pm x_{c}$, while $\left|K_{z}(x)\right|>K_{c}$ in the vortex-free region $-b<x<b$. Shown are results for $I_{0}=1.545 I_{c 1}$ when $b=0.600$ and $a=1.077$ (dotted curves), $I_{0}=1.683 I_{c 1}$ when $b=0.400$ and $a=1.337$ (short-dashed curves), $I_{0}=1.966 I_{c 1}$ when $b=0.200$ and $a=1.630$ (medium-dashed curves), and $I_{0}=2 I_{c 1}+I_{c 0}=2.500 I_{c 1}$ when $b=0$ and $a=1.933$ (longdashed curves). For $I_{0} \geq 2 I_{c 1}+I_{c 0}, K_{z}(x)=-K_{c}$ in the region $-a<x<a$ and $\left|\bar{K}_{z}(x)\right|<K_{c}$ outside this region.

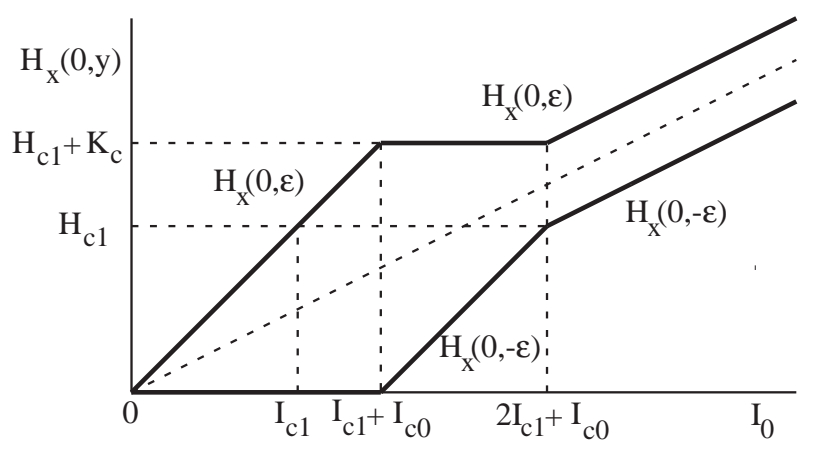

FIG. 14: Parallel magnetic fields at $x=0$ at the top and bottom surfaces $H_{x}(0, \epsilon)$ and $H_{x}(0,-\epsilon)$ vs $I_{0}$ in the presence of bulk pinning, shown here for $K_{c}=H_{c 1} / 2$, such that $I_{c 1}+I_{c 0}=1.5 I_{c 1}$ and $2 I_{c 1}+I_{c 0}=2.5 I_{c 1}$. When $0 \leq I_{0} \leq I_{c 1}+I_{c 0}, H_{x}(0, \epsilon)=H_{M x}(0, \epsilon)[$ Eq. (9)] and $H_{x}(0,-\epsilon)=H_{M x}(0,-\epsilon)=0$. When $I_{c 1}+I_{c 0} \leq I_{0} \leq$ $2 I_{c 1}+I_{c 0}, H_{x}(0, \epsilon)=H_{M x}(0, \epsilon)+H_{v x}(0, \epsilon)=H_{c 1}+\bar{K}_{c}$, and $H_{x}(0,-\epsilon)=H_{v x}(0,-\epsilon)=-H_{v x}(0, \epsilon)$. When $I_{0} \geq 2 I_{c 1}+I_{c 0}$, $H_{x}(0, \pm \epsilon)=H_{0 x}(0,0) \pm K_{c} / 2$.

Figure 14 shows plots of $H_{x}(0, \pm \epsilon)=H_{M x}(0, \pm \epsilon)+$ $H_{v x}(0, \pm \epsilon)$ vs $I_{0}$. For $0 \leq I_{0} \leq I_{c 1}+I_{c 0}$, we have $H_{x}(0, \epsilon)=H_{M x}(0, \epsilon)=H_{c 1} I_{0} / I_{c 1}$ and $H_{x}(0,-\epsilon)=$ $H_{M x}(0,-\epsilon)=0$. For $I_{c 1}+I_{c 0} \leq I_{0} \leq 2 I_{c 1}+I_{c 0}$, we have $H_{x}(0, \epsilon)=H_{M x}(0, \epsilon)+H_{v x}(0, \epsilon)=H_{c 1}+K_{c}$ and $H_{x}(0,-\epsilon)=H_{v x}(0,-\epsilon)$, where $H_{M x}(0, \epsilon)=H_{c 1} I_{0} / I_{c 1}$ and $H_{v x}(0, \pm \epsilon)=\mp H_{c 1}\left(I_{0}-I_{c 1}-I_{c 0}\right) / I_{c 1}$. When $I_{0}=$ $2 I_{c 1}+I_{c 0}, H_{x}(0, \epsilon)=H_{c 1}+K_{c}$ and $H_{x}(0,-\epsilon)=H_{c 1}$. For $I_{0} \geq 2 I_{c 1}+I_{c 0}, H_{x}(0, \pm \epsilon)=H_{0 x}(0,0) \pm K_{c} / 2=$

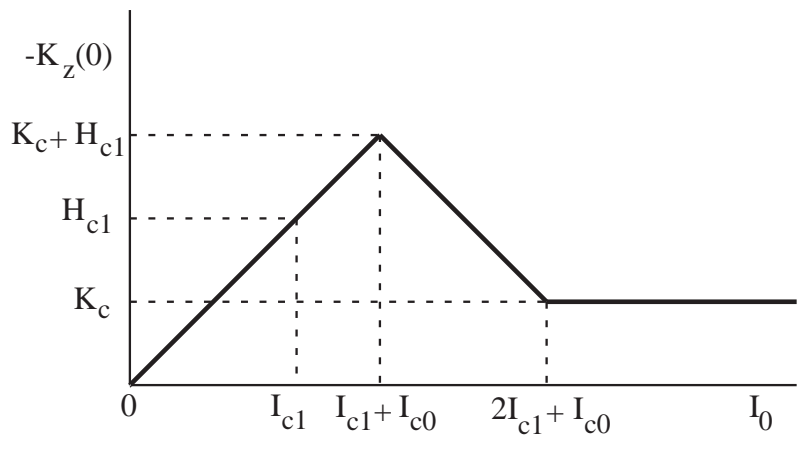

FIG. 15: Magnitude of the sheet-current density at the origin $-K_{z}(0)=H_{x}(0, \epsilon)-H_{x}(0,-\epsilon)$ vs $I_{0}$ in the presence of weak bulk pinning, shown here for $K_{c}=H_{c 1} / 2$, such that $I_{c 2}=$ $2 I_{c 1}-I_{c 0}=1.5 I_{c 1}$ (see Fig. 14).

$H_{c 1} I_{0} / 2 I_{c 1} \pm K_{c} / 2$.

Figure 15 shows a plot of $-K_{z}(0)$ vs $I_{0}$, where $K_{z}(0)=$ $H_{x}(0,-\epsilon)-H_{x}(0, \epsilon)$. For $0 \leq I_{0} \leq I_{c 1}+I_{c 0},-K_{z}(0)=$ $\left(H_{c 1}+K_{c}\right) I_{0} /\left(I_{c 1}+I_{c 0}\right)$, and for $I_{c 1}+I_{c 0} \leq I_{0} \leq 2 I_{c 1}+I_{c 0}$, $-K_{z}(0)=K_{c}+H_{c 1}\left(2 I_{c 1}+I_{c 0}-I_{0}\right) / I_{c 1}$. For $I_{0} \geq 2 I_{c 1}+$ $I_{c 0},-K_{z}(0)=K_{c}$.

The vortex-generated complex potential $\mathcal{G}_{v}(\zeta)=$ $\mathcal{G}(\zeta)-\mathcal{G}_{M}(\zeta)$ [see Eqs. (10) and [B14] ] in the presence of weak bulk pinning $\left(0<K_{c}<H_{c 1}\right)$ is, when $I_{c 1}<I_{0}<I_{c 2}$, such that $0<b<a$,

$$
\mathcal{G}_{v}(\zeta)=\frac{I_{0}}{2 \pi}\left[g_{v}\left(\zeta, y_{1}\right)-g_{v}\left(\zeta, y_{2}\right)\right] \pm i \frac{K_{c}}{2} \zeta,
$$

where $g_{v}(\zeta, y)$ is given in Eq. (28). Shown in Fig. 16 is a contour plot of the real part of $\mathcal{G}_{v}(x+i y)$. These contours correspond to the magnetic field lines of the vortex-generated magnetic field. The magnetic field flows in a generally counterclockwise direction, carried by vortices in the region $b<x<a$ up through the film and by antivortices in the region $-a<x<-b$ back down through the film. A contour plot of the real part of $\mathcal{G}(x+i y)=\mathcal{G}_{M}(x+i y)+\mathcal{G}_{v}(x+i y)$ would also show the magnetic field lines generated by the two wires, as in Fig. 2.

When $I_{0}>2 I_{c 1}+I_{c 0}$, the gap between the vortex dome and the antivortex dome is closed $(b=0)$, and the magnetic-field distribution thus can be characterized as a dome of vortices in the region $0<x<a$ carrying magnetic flux up through the film and an adjacent dome of antivortices in the region $-a<x<0$ carrying an equal amount of magnetic flux back down through the film. The outer boundaries of these domes $( \pm a)$ depend upon the values of $I_{0}$ and $K_{c}$, and the magnetic-field and sheetcurrent-density distributions can be calculated as follows. The complex magnetic field $\mathcal{H}(\zeta)=\mathcal{H}_{M}(\zeta)+\mathcal{H}_{v}(\zeta)$ is as given in Eq. (B13), except that $b=0$ and $\phi(\zeta), s_{1}$, and $s_{2}$ are now given by Eqs. (B19), (B20), and (B21). Similarly, the vortex-generated complex magnetic field $\mathcal{H}_{v}(\zeta)$ is as given in Eq. (32), and $H_{v y}(x, 0), H_{v x}(x, \epsilon)=$ $-H_{v x}(x,-\epsilon)$, and $K_{v z}(x)=H_{v x}(x,-\epsilon)-H_{v x}(x, \epsilon)$ are as given in Eqs. (33), (34), and (35), except that now just 


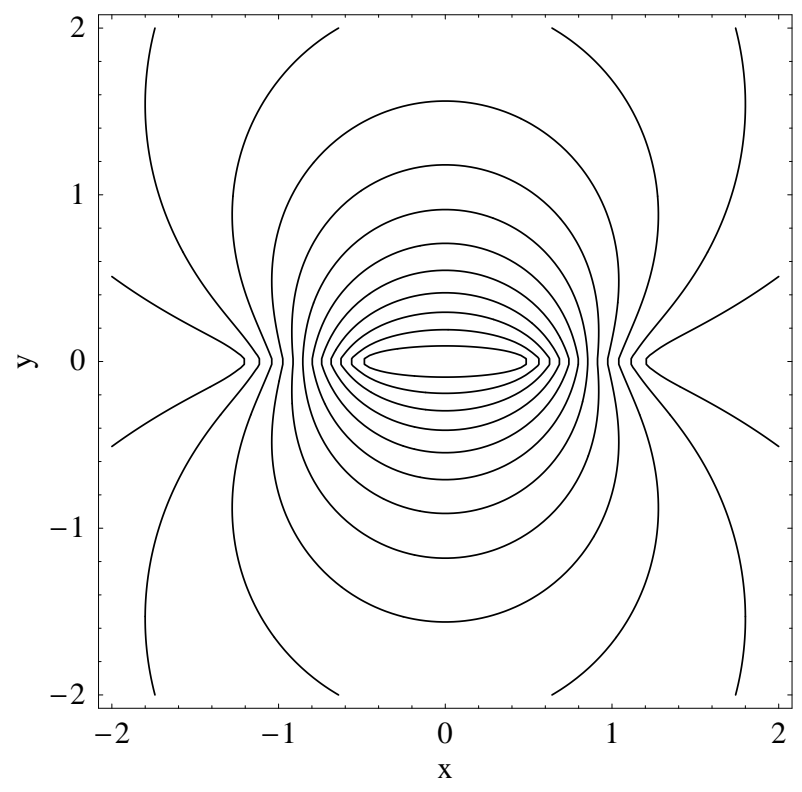

FIG. 16: Contour plot of the real part of the vortex-generated complex potential in the presence of weak bulk pinning [Eq. (40)] $\mathcal{G}_{v}(x+i y)$ vs $x$ and $y$ for $y_{1}=1, y_{2}=2$, $K_{c}=H_{c 1} / 2, I_{0}=1.683 I_{c 1}, a=1.337$, and $b=0.4$, as for the short-dashed curves in Figs. 12 and 13. The contours correspond to magnetic field lines of the vortex-generated complex field $\mathcal{H}_{v}(x+i y)$ given in Eq. (32).

above or below the real axis,

$$
\phi(x \pm i \epsilon)= \begin{cases}\tilde{\phi}(x), & 0<|x|<a \\ \pm i \tilde{\phi}(x), & |x|>a\end{cases}
$$

where

$$
\tilde{\phi}(x)= \begin{cases}x \sqrt{a^{2}-x^{2}}, & 0<|x|<a, \\ -|x| \sqrt{x^{2}-a^{2}}, & |x|>a .\end{cases}
$$

For given values of $I_{0}$ and $K_{c}$ the value of $a$ in the above equations is determined by Eq. (B25), which follows from the requirement that $\int_{-\infty}^{+\infty} K_{z}(x) d x=0$, and by Eq. (36) (but with $b=0$ ), which follows from the requirement that $H_{x}(0, \epsilon)=H_{c 1}+K_{c}$. Numerical solutions for $a$ obtained in this way are shown in Fig. [1] vs $I_{0} / I_{c 1}$ for $I_{0}>2 I_{c 1}+$ $I_{c 0}$ (where $b=0$ ) for the case of $y_{1}=1$ and $y_{2}=2$. Figures 17 and 18 show plots of $H_{y}(x, 0)=H_{v y}(x, 0)$ and $K_{z}(x)$ vs $x$ for several values of $I_{0} \geq 2 I_{c 1}+I_{c 0}$ when $K_{c}=H_{c 1} / 2$.

When $I_{0}>2 I_{c 1}+I_{c 0}$, such that $a>0$ and $b=0$, the vortex-generated complex potential $\mathcal{G}_{v}(\zeta)=\mathcal{G}(\zeta)-$ $\mathcal{G}_{M}(\zeta)$ [see Eqs. (10), (B14), and (B22)] in the presence of bulk pinning is

$$
\mathcal{G}_{v}(\zeta)=\frac{I_{0}}{2 \pi}\left[g_{v}\left(\zeta, y_{1}\right)-g_{v}\left(\zeta, y_{2}\right)\right] \pm i \frac{K_{c}}{2} \zeta
$$

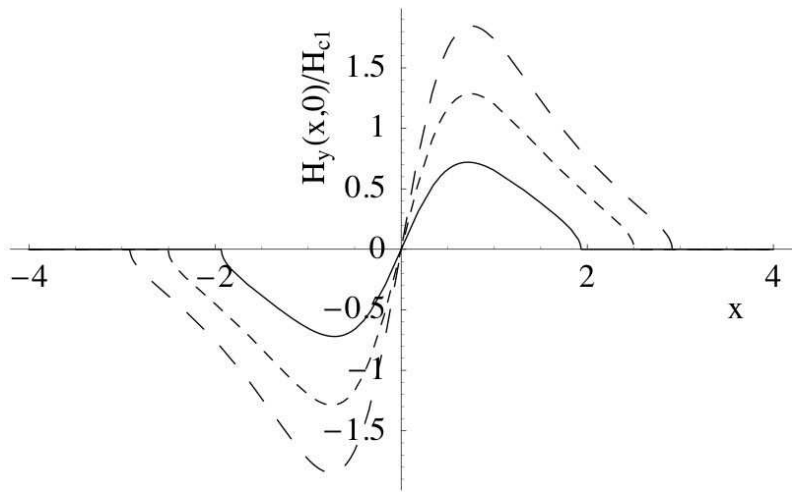

FIG. 17: When $I_{0} \geq 2 I_{c 1}+I_{c 0}$ in the presence of bulk pinning, the perpendicular magnetic field $H_{y}(x, 0) / H_{c 1}=$ $H_{v y}(x, 0) / H_{c 1}$ vs $x$ exhibits adjacent vortex and antivortex flux domes at $0<|x|<a$, as shown here for $K_{c}=H_{c 1} / 2$, $I_{c 0}=0.5 I_{c 1}, y_{1}=1$, and $y_{2}=2$. As $I_{0}$ increases, the domes become taller and wider, as shown for $I_{0}=2.5 I_{c 1}$ when $a=1.933$ (solid curves), $I_{0}=4.25 I_{c 1}$ when $a=2.504$ (shortdashed curves), and $I_{0}=6 I_{c 1}$ when $a=2.913$ (long-dashed curves).

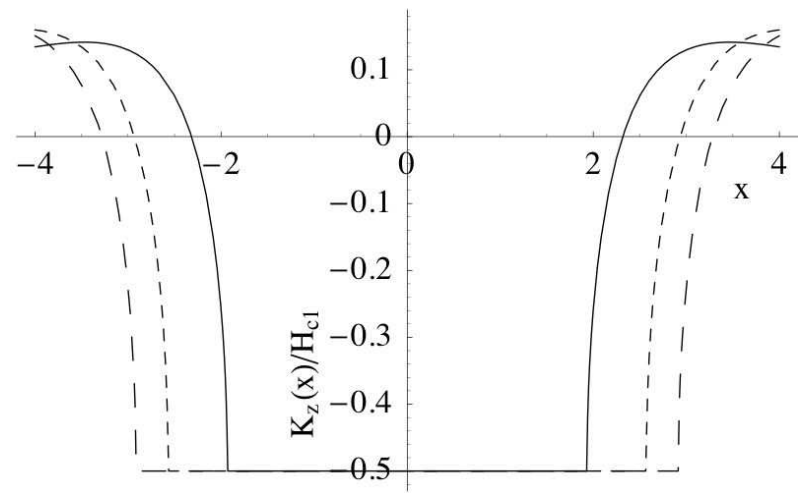

FIG. 18: Sheet-current density $K_{z}(x, 0) / H_{c 1}$ vs $x$ when $I_{0} \geq$ $2 I_{c 1}+I_{c 0}$ in the presence of bulk pinning, shown here for $K_{c}=$ $H_{c 1} / 2, I_{c 0}=0.5 I_{c 1}, y_{1}=1$ and $y_{2}=2$. Shown are results for $I_{0}=2.5 I_{c 1}$ when $a=1.933$ (solid curve), $I_{0}=4.25 I_{c 1}$ when $a=2.504$ (short-dashed curve), and $I_{0}=6 I_{c 1}$ when $a=2.913$ (long-dashed curve), corresponding to the cases shown in Fig. 17

where

$$
\begin{aligned}
g_{v}(\zeta, y)= & \mp i \arctan (\zeta / y)+\sqrt{\frac{a^{2}-\zeta^{2}}{a^{2}+y^{2}}}-\frac{a}{\sqrt{a^{2}+y^{2}}} \\
& -\operatorname{arctanh} \sqrt{\frac{a^{2}-\zeta^{2}}{a^{2}+y^{2}}}+\operatorname{arctanh} \frac{a}{\sqrt{a^{2}+y^{2}}},
\end{aligned}
$$

and the upper (lower) signs hold in the upper (lower) half $\zeta$ plane. Shown in Fig. [19] is a contour plot of the real part of $\mathcal{G}_{v}(x+i y)$. These contours correspond to the magnetic field lines of the vortex-generated magnetic field. The magnetic field flows in a generally counterclockwise direction, carried by vortices in the region $0<x<a$ up through the film and by antivortices in the region $-a<x<0$ back down through the film. A contour plot 


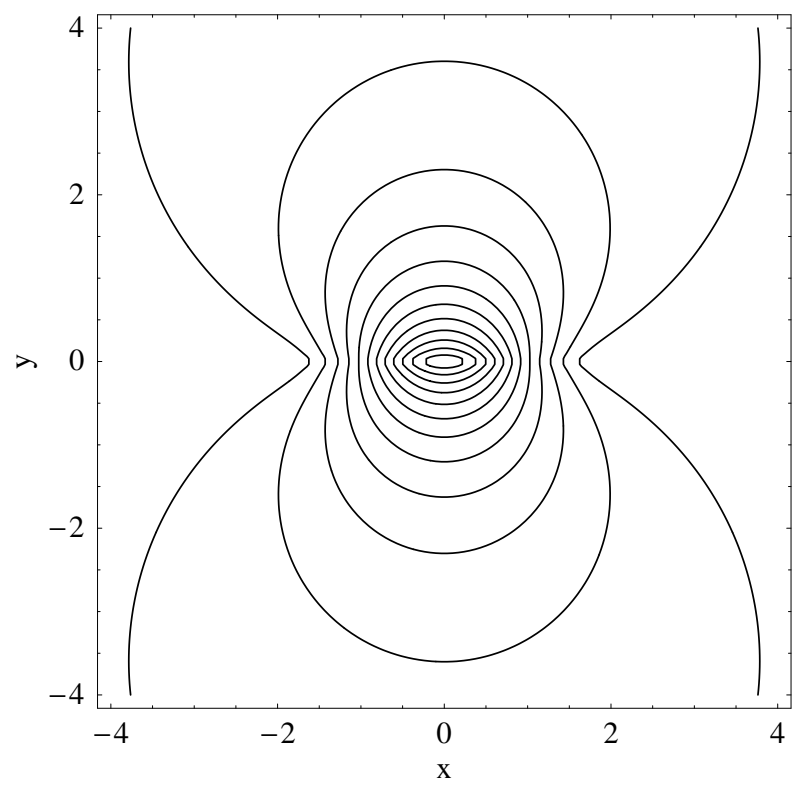

FIG. 19: Contour plot of the real part of the vortex-generated complex potential in the presence of bulk pinning [Eq. 43] ] $\mathcal{G}_{v}(x+i y)$ vs $x$ and $y$ for $y_{1}=1, y_{2}=2, K_{c}=H_{c 1} / 2$, $I_{0}=2 I_{c 1}+I_{c 0}=2.5 I_{c 1}, a=1.933$, and $b=0$, as for the solid curves in Figs. 17] and 18 The contours correspond to magnetic field lines of the vortex-generated complex field $\mathcal{H}_{v}(x+i y)$ given by Eq. (32).

of the real part of $\mathcal{G}(x+i y)=\mathcal{G}_{M}(x+i y)+\mathcal{G}_{v}(x+i y)$ would also show the magnetic field lines generated by the two wires, as in Fig. 2.

\section{E. Flux domes in the presence of strong bulk pinning}

We consider here briefly the case of superconducting films in which bulk pinning, characterized by a fieldindependent critical sheet-current density $K_{c}=j_{c} d>0$, is so strong that $K_{c} \gg H_{c 1}$. In principle, the process of vortex entry is qualitatively the same as discussed in Sec. IID However, in the limit that $H_{c 1} / K_{c} \rightarrow 0$, the width of the current region $I_{c 1}+I_{c 0}$ to $2 I_{c 1}+I_{c 0}$ (in which there is a gap of width $2 b$ between the vortex and antivortex domes) shrinks to zero, and $I_{c 0}$ becomes the only critical current of practical interest. Essentially, as soon as $I_{0}$ exceeds $I_{c 0}$, the vortices penetrating from the top surface divide in such a way as to produce adjacent vortex and antivortex flux domes in the regions $0<x<a$ and $-a<x<0$, and the sheet-current density is $K_{z}(x)=-K_{c}$ in these regions. The perpendicular magnetic field $H_{y}(x, 0)$, sheet-current density $K_{z}(x)$ and vortex-generated complex potential $\mathcal{G}_{v}(x+i y)$ can be calculated as discussed in Sec. IID for the case that $b=0$, and plots of all these quantities look very similar to those in Figs. 17] 18, and 19.

Figure 20 shows plots of $H_{x}(0, \pm \epsilon)=H_{M x}(0, \pm \epsilon)+$

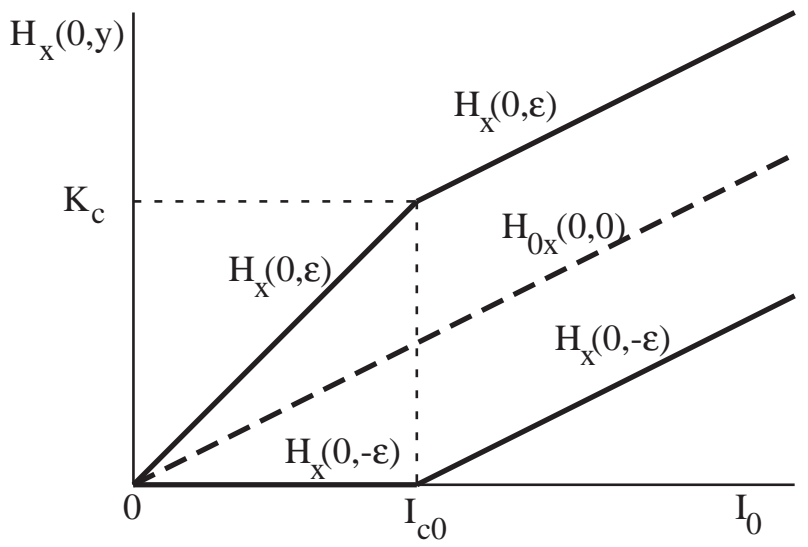

FIG. 20: Parallel magnetic fields at $x=0$ at the top and bottom surfaces $H_{x}(0, \epsilon)$ and $H_{x}(0,-\epsilon)$ vs $I_{0}$ for strong bulk pinning in the limit as $H_{c 1} / K_{c} \rightarrow 0$. When $0 \leq I_{0} \leq I_{c 0}$, $H_{x}(0, \epsilon)=H_{M x}(0, \epsilon)[$ Eq. (8)] $]$ and $H_{x}(0,-\epsilon)=H_{M x}(0,-\epsilon)=$ 0 . When $I_{0} \geq I_{c 0}, H_{x}(0, \pm \epsilon)=H_{0 x}(0,0) \pm K_{c} / 2$ [see Eq. (2)].

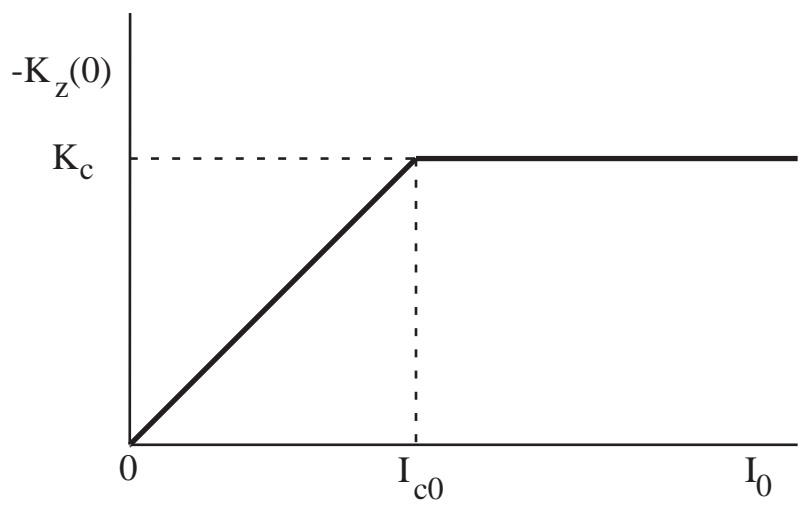

FIG. 21: Magnitude of the sheet-current density at the origin $-K_{z}(0)=H_{x}(0, \epsilon)-H_{x}(0,-\epsilon)$ vs $I_{0}$ for strong bulk pinning in the limit as $H_{c 1} / K_{c} \rightarrow 0$ (see Fig. 20).

$H_{v x}(0, \pm \epsilon)$ vs $I_{0}$ in the limit $H_{c 1} / K_{c} \rightarrow 0 . \quad$ For $0 \leq I_{0} \leq I_{c 0}$, we have $H_{x}(0, \epsilon)=H_{M x}(0, \epsilon)=K_{c} I_{0} / I_{c 0}$ and $H_{x}(0,-\epsilon)=H_{M x}(0,-\epsilon)=0$. For $I_{0} \geq I_{c 0}$, $H_{x}(0, \pm \epsilon)=H_{0 x}(0,0) \pm K_{c} / 2=K_{c} I_{0} / 2 I_{c 0} \pm K_{c} / 2$. Figure 21 shows a plot of $-K_{z}(0)$ vs $I_{0}$ in the same limit, where $K_{z}(0)=H_{x}(0,-\epsilon)-H_{x}(0, \epsilon)$. For $0 \leq I_{0} \leq I_{c 0}$, $-K_{z}(0)=K_{c} I_{0} / I_{c 0}$, and for $I_{0} \geq I_{c 0},-K_{z}(0)=K_{c}$.

\section{SUMMARY AND DISCUSSION}

In this paper we have predicted that separated vortex and antivortex flux domes can be produced in weakpinning type-II superconducting films subjected to local magnetic fields generated by current-carrying wires above the film's surface and far from the edges. To calculate these effects analytically, we have chosen an idealized geometry of two parallel infinitely long wires above an infinite superconducting film. However, the basic phenomenon of the creation of separated vortex and antivor- 
tex flux domes without nucleation at the film's edges is far more general than in the geometry we have considered here.

For example, consider the case of a bulk-pinning-free superconducting film of finite size in the $x z$ plane and a small coil at $(x, y, z)=\left(0, y_{0}, 0\right)$ a short distance above the film. Suppose the coil produces a magnetic dipole moment $\boldsymbol{m}=-\hat{x} m$. When $m$ is small, the film remains in the Meissner state, and if the magnetic field below the film is very small, the coil-generated dipole magnetic field and its image produce a magnetic field at the film's top surface $\boldsymbol{H}_{M}=\hat{x} H_{M x}+\hat{z} H_{M z}$, where $H_{M x}(x, \epsilon, z)=$ $2 m\left(y_{0}^{2}-2 x^{2}+z^{2}\right) / R_{0}^{5}, H_{M z}(x, \epsilon, z)=-6 m x z / R_{0}^{5}$, and $R_{0}=\sqrt{x^{2}+y_{0}^{2}+z^{2}}$. The induced sheet-current density is $\boldsymbol{K}_{M}=\hat{x} K_{M x}+\hat{z} K_{M z}$, where $K_{M x}(x, z)=$ $H_{M z}(x, \epsilon, z)$ and $K_{M z}(x, z)=-H_{M x}(x, \epsilon, z)$, such that $\nabla \cdot \boldsymbol{K}_{M}=0$. In the plane $z=0$ the magnetic-field and sheet-current distributions resemble those shown in Figs. 2 and 3. The maximum magnetic field parallel to the top surface occurs at the origin, where $\boldsymbol{H}_{M}(0, \epsilon, 0)=\left(2 m / y_{0}^{3}\right) \hat{x}$, and the first flux penetration through the film occurs when $m$ increases to the value $m_{c}=H_{c 1} y_{0}^{3} / 2$. The penetrating vortex splits into a vortex and an antivortex, and the vortex is driven by the Meissner screening currents via the corresponding Lorentz force $\boldsymbol{F}(x, z)=\phi_{0} \boldsymbol{H}_{M}(x, \epsilon, z)$ to the point $(x, y, z)=\left(y_{0} / \sqrt{2}, 0,0\right)$, while the antivortex is similarly driven to $(x, y, z)=\left(-y_{0} / \sqrt{2}, 0,0\right)$. A further increase in the coil's current $\left(m>m_{c}\right)$ will result in more nucleating vortices and antivortices and cause the development of vortex and antivortex flux domes centered at $(x, y, z)=\left( \pm y_{0} / \sqrt{2}, 0,0\right)$. Vortex and antivortex flux domes also could be produced by bringing a permanent magnet with magnetic dipole moment $\boldsymbol{m}=-\hat{x} m$ close to the film.

Similar effects should occur in superconducting films with bulk pinning. In particular, when the maximum magnetic field parallel to the surface, accounting for image fields, exceeds $H_{c 1}+K_{c}$, vortex and antivortex flux domes should be produced with properties similar to those described in Secs. IID and IIE In the above geometry with a small coil or a permanent magnet producing a magnetic moment $\boldsymbol{m}=-\hat{x} m$, we expect that the main effect of bulk pinning will be to reduce the separation between the vortex and antivortex flux domes.

In this paper we have confined our attention to the case in which an initially flux-free film is subjected to locally applied magnetic fields increasing in magnitude. We expect that interesting hysteretic effects, similar to those in a finite-width film with a geometrical barrier, 16 will occur when ac magnetic fields are applied. For example, consider a bulk-pinning-free superconducting film in the geometry studied in Sec. [II but with $I_{0}$ being cycled. As $I_{0}$ is increased from zero, we expect vortex and antivortex domes to develop as predicted in Sec.[IC where the dome boundaries $a$ and $b$ are determined in part by the condition that $H_{x}(0, \epsilon)=H_{c 1}$ [Eq. (25)]. Suppose that these values are $a_{\max }$ and $b_{\max }$ when $I_{0}$ increases to some maximum value $I_{\max }$, where $I_{c 1}<I_{\max }<2 I_{c 1}$, $a_{\max }=x_{0}^{2} / b_{\max }>x_{0}$, and $b_{\max }<x_{0}=\sqrt{y_{1} y_{2}}$. If $I_{0}$ is now decreased, the condition that $H_{x}(0, \epsilon)=H_{c 1}$ is replaced by the condition that the magnetic flux under each flux dome remains constant; i.e., $\operatorname{Re}[\mathcal{G}(a)-\mathcal{G}(b)]$ $=$ const. As a result, as $I_{0}$ decreases, the width of each dome increases while its height decreases; i.e., $b$ decreases $\left(b<b_{\max }\right)$ and $a$ increases $\left(a>a_{\max }\right)$. The resulting values of $b$ and $a$ can be calculated as functions of $I_{0}$ using the relations $a=x_{0}^{2} / b$ and

$$
I_{0} \Delta g(a, b)=I_{\max } \Delta g\left(a_{\max }, b_{\max }\right),
$$

where [see Eqs. (B14) and (B15)]

$$
\begin{aligned}
\Delta g(a, b)= & \operatorname{Re}\left\{\left[g_{0}\left(a, y_{1}\right)-g_{0}\left(a, y_{2}\right)\right]\right. \\
& \left.-\left[g_{0}\left(b, y_{1}\right)-g_{0}\left(b, y_{2}\right)\right]\right\} .
\end{aligned}
$$

So long as $b>0$, vortex-antivortex annihilation cannot occur because the sheet current flowing in the region $|x|<b$ still keeps vortices and antivortices apart. In fact, a subsequent increase of $I_{0}$ would produce reversible changes in the width and height of the vortex and antivortex domes, provided $I_{0}<I_{\max }$. However, the vortex-free gap of width $2 b$ closes when $I_{0}$ is decreased to the value $I_{e x}$ at which $b \rightarrow 0$ and $a \rightarrow \infty$, where

$$
I_{e x}=I_{\max } \Delta g\left(a_{\max }, b_{\max }\right) / \Delta g(\infty, 0)
$$

and $\Delta g(\infty, 0)=\ln \left(y_{2} / y_{1}\right)$. When $I_{0}=I_{e x}$, the sheetcurrent density becomes everywhere zero, the film appears as if it were completely incapable of screening, and the magnetic field distribution (averaged over a length of the order of the intervortex separation) is essentially the same as it would be in the absence of the film. On the other hand, viewing the field distribution as a linear superposition of the Meissner-state response and a vortex-antivortex distribution, we see that when the gap of width $2 b$ closes, vortex-antivortex annihilation begins to occur at $x=0$, and magnetic flux begins to exit from the vortex and antivortex domes. As $I_{0}$ decreases from $I_{e x}$ to zero, the magnitude of the magnetic flux under each dome decreases to zero. When $I_{0}=0$, the film is again flux-free, and as $I_{0}$ further decreases to $-I_{\max }$, the behavior is very similar to that for increasing $I_{0}$, except that the roles of vortices and antivortices are interchanged.

We expect that similar but somewhat more complicated hysteretic effects will occur in the presence of bulk pinning. In the case of strong bulk pinning $\left(K_{c} \gg H_{c 1}\right)$, the role of $H_{c 1}$ can be neglected, and the hysteretic properties can be calculated analytically as in Ref. 25, which treats the response of a superconducting film to currents in linear wires in arrangements similar to that discussed in Sec. III Such calculations illuminate the fundamental physics underlying the ac technique introduced by Claassen et $a l^{26}$ to determine the critical current density $j_{c}$ in superconducting films. This technique employs a small coil, placed just above the film, carrying a sinusoidal current. When the current amplitude exceeds the 
value at which the maximum induced sheet-current density reaches $K_{c}=j_{c} d$, a third-harmonic voltage appears in the coil. 26.27.28.29 A similar technique was introduced by Hochmuth and Lorenz 30

The effects discussed in this paper and applied to typeII superconducting films are quite general and also should be observed in type-I superconductors. Magnetic flux domes consisting of intermediate-state regions containing multiply quantized flux tubes have been observed in type-I strips in which the geometric barrier plays a dominant role $\frac{9}{-}$ It is therefore likely that separated domes of positive and negative magnetic flux produced in response to nearby current-carrying wires, coils, or permanent magnets also will be observed in weak-pinning type-I superconducting films, foils, or plates when the net parallel field at the surface exceeds the bulk thermodynamic critical field $H_{c}$ (or, when bulk pinning is present, $\left.H_{c}+K_{c}\right)$. In type-I superconductors, however, we expect that the magnetic flux will enter in the form of the intermediate state. The analog of a vortex dome will be an intermediate-state region consisting either of an array of multiply quantized flux tubes or a meandering normal-superconducting domain structure carrying magnetic flux up through the film, while the analog of an antivortex dome will be a similar intermediate-state region carrying magnetic flux down through the film. Such magnetic structures should be observable by magneto-optics or related means by placing the magnetic-field source on one side of the sample and the magnetic-field detector on the opposite side.

\section{ACKNOWLEDGMENTS}

We thank V. G. Kogan for stimulating discussions. This manuscript has been authored in part by Iowa State University of Science and Technology under Contract No. W-7405-ENG-82 with the U.S. Department of Energy.

\section{APPENDIX A: SCREENING EFFECTS}

The primary purpose of this paper is to describe in detail how magnetic flux penetrates through a superconducting film in the form of vortices and antivortices, using the assumption that the magnetic field below the film is initially negligibly small. In the following we present results confirming that this is an excellent approximation when $d>\lambda$ and $d \ll y_{1}$.

Consider the experimental configuration shown in Fig. 1 but allow for the finite thickness $d$ of the superconducting film in the region $|z|<d / 2$. When the film, characterized by the London penetration depth $\lambda$, is in the Meissner state, the vector potential and the magnetic field throughout all space, as well as the supercurrent density in the film, can be calculated as described in Ref. 31. The results for the $x$ components of the magnetic fields $H_{x}(0, \mp d / 2)$ at the bottom and top surfaces of the film, expressed in units of $H_{0 x}(0,0)$ [Eq. (2)], are

$$
\begin{aligned}
& \frac{H_{x}(0,-d / 2)}{H_{0 x}(0,0)}=\frac{2 y_{1} y_{2}}{\left(y_{2}-y_{1}\right)} \int_{0}^{\infty}\left[\frac{Q q}{2 q Q \cosh (Q d)+\left(q^{2}+Q^{2}\right) \sinh (Q d)}\right]\left(e^{-q y_{1}}-e^{-q y_{2}}\right) e^{q d / 2} d q, \\
& \frac{H_{x}(0,+d / 2)}{H_{0 x}(0,0)}=\frac{2 y_{1} y_{2}}{\left(y_{2}-y_{1}\right)} \int_{0}^{\infty}\left[\frac{Q[q \cosh (Q d)+Q \sinh (Q d)]}{2 q Q \cosh (Q d)+\left(q^{2}+Q^{2}\right) \sinh (Q d)}\right]\left(e^{-q y_{1}}-e^{-q y_{2}}\right) e^{q d / 2} d q,
\end{aligned}
$$

where $Q=\sqrt{q^{2}+\lambda^{-2}}$. These quantities are plotted as the solid curves in Figs. 22] and 23] as functions of $\lambda / d$ for the case that $d=y_{1} / 1000$ and $y_{2}=2 y_{1}$.

When $\lambda \rightarrow 0$, the film screens perfectly, such that $H_{x}(0,-d / 2)=0$ and $H_{x}(0,+d / 2)=2 H_{x 0}(0, d / 2)$. In the opposite limit, when $\lambda \rightarrow \infty, H_{x}(0,-d / 2)=$ $H_{x 0}(0,-d / 2)$ and $H_{x}(0, d / 2)=H_{x 0}(0, d / 2)$, where $H_{x 0}(0, y)=I_{0}\left(y_{2}-y_{1}\right) / 2 \pi\left(y_{1}-y\right)\left(y_{2}-y\right)$ is the magnetic field produced by the wires in the plane $x=0$ for $y<y_{1}$ in the film's absence.

Equations (A1) and (A2) reduce to simpler expressions when $d \ll y_{1}$ and either $\lambda<d$ or, if $\lambda>d$, the Pearl length ${ }^{32} \Lambda=\lambda^{2} / d$ obeys $\Lambda \ll y_{1}$. Then to good approximation $q$ can be set equal to zero inside the brackets and
$Q$ can be replaced by $1 / \lambda$. The resulting integrals yield

$$
\begin{aligned}
& \frac{H_{x}(0,-d / 2)}{H_{0 x}(0,0)}=\frac{2\left(y_{1}+y_{2}\right) \lambda}{y_{1} y_{2} \sinh (d / \lambda)} \\
& \frac{H_{x}(0,+d / 2)}{H_{0 x}(0,0)}=2-\frac{2\left(y_{1}+y_{2}\right) \lambda}{y_{1} y_{2} \tanh (d / \lambda)} .
\end{aligned}
$$

As shown by the long-dashed curves in Figs. 22 and 23 these expressions are excellent approximations, indistinguishable from the solid curves, when $\lambda / d<1$ or $\Lambda \ll y_{1}$ when $\lambda / d>1$. However, the long-dashed curves deviate significantly from the solid curves for $\lambda / d>10$, which is to be expected, since for the parameters used for the figures, $\Lambda \approx y_{1}$ when $\lambda / d \approx 30$.

To evaluate Eqs. A1 and A2 for all values of the Pearl length $32=\lambda^{2} / d$ when $d \ll y_{1}$ and $\lambda \gg d$, it is a good approximation to ignore $q^{2}$ relative to $1 / \lambda^{2}$ 


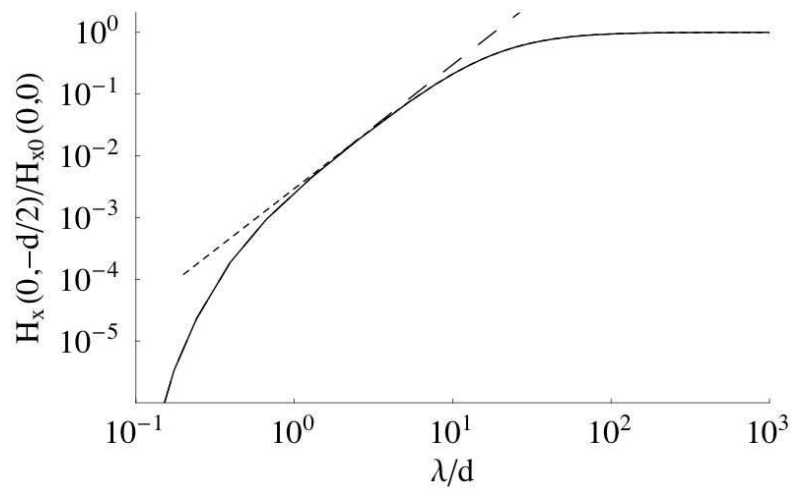

FIG. 22: Magnetic field just below the film $H_{x}(0,-d / 2) / H_{x 0}(0,0)$ vs $\lambda / d$, shown here for $d=y_{1} / 1000$ and $y_{2}=2 y_{1}$ as calculated from Eq. (A1) (solid curve), Eq. A3 (long-dashed curve), and Eq. A5 (short-dashed curve).

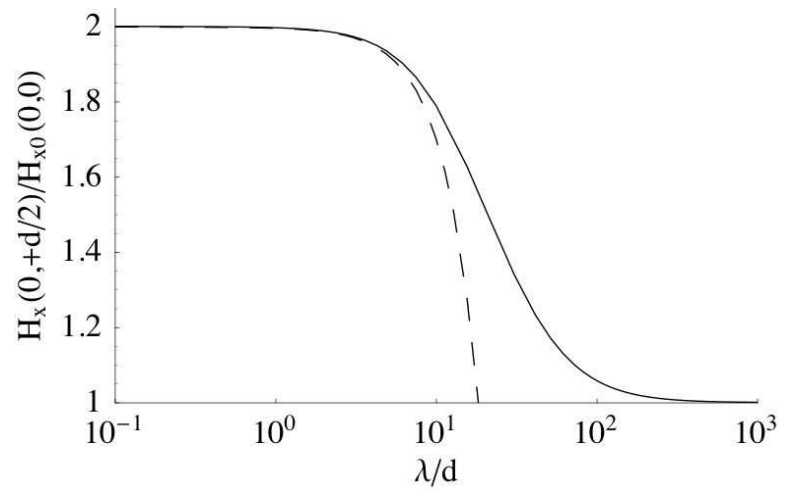

FIG. 23: Magnetic field just above the film $H_{x}(0,+d / 2) / H_{x 0}(0,0)$ vs $\lambda / d$, shown here for $d=y_{1} / 1000$ and $y_{2}=2 y_{1}$ as calculated from Eq. A2 (solid curve) and Eq. (A4) (dashed curve). A plot of the same quantity using Eq. (A6) would be indistinguishable from the solid curve.

inside the brackets and to replace $Q$ by $1 / \lambda$ but to retain the terms proportional to $q$ in the denominators. This approximation is equivalent to the assertion that when $d \ll \lambda$, the only length that determines the screening properties of the film is $\Lambda$. This procedure yields the following approximate results:

$$
\begin{aligned}
& H_{x}(0,-d / 2) / H_{0 x}(0,0)=1-I \\
& H_{x}(0,+d / 2) / H_{0 x}(0,0)=1+I
\end{aligned}
$$

where

$$
\begin{gathered}
I=\frac{y_{1} y_{2}}{\left(y_{2}-y_{1}\right)} \int_{0}^{\infty} \frac{1}{1+2 q \Lambda}\left(e^{-q y_{1}}-e^{-q y_{2}}\right) d q, \\
=\frac{y_{1} y_{2}}{2\left(y_{2}-y_{1}\right) \Lambda}\left[e^{y_{2} / 2 \Lambda} \operatorname{Ei}\left(-y_{2} / 2 \Lambda\right)\right. \\
\left.\quad-e^{y_{1} / 2 \Lambda} \operatorname{Ei}\left(-y_{1} / 2 \Lambda\right)\right]
\end{gathered}
$$

and $\operatorname{Ei}(x)$ is the exponential integral. On the scale of Fig. 22] the approximation for $H_{x}(0,-d / 2)$ given in
Eq. (A5), shown as the short-dashed curve, is indistinguishable from the solid curve for values of $\lambda / d>3$. Surprisingly, on the scale of Fig. 23] the approximation for $H_{x}(0, d / 2)$ given in Eq. A6 in indistinguishable from the solid curve for all values of $\lambda / d$.

\section{APPENDIX B: COMPLEX FIELD AND COMPLEX POTENTIAL}

In the following we derive the complex field $\mathcal{H}(\zeta)$ and complex potential $\mathcal{G}(\zeta)$ satisfying the boundary conditions that the perpendicular $(y)$ component of the magnetic field in the plane of the film obeys $H_{y}(x, 0)=0$ for $|x|<b$ or $|x|>a$ and that the sheet-current density in the $z$ direction obeys $K_{z}(x)=-K_{c}$ for $b<|x|<a$. We begin by considering the function

$$
F(\zeta)=\left[\mathcal{H}(\zeta)-\mathcal{H}_{\|}\right] / \phi(\zeta)
$$

where $\mathcal{H}(\zeta)$ is defined in Eq. (1),

$$
\mathcal{H}_{\|}(\zeta)=i \frac{I_{0}}{2 \pi}\left(\frac{y_{1}}{\zeta^{2}+y_{1}^{2}}-\frac{y_{2}}{\zeta^{2}+y_{2}^{2}}\right),
$$

and

$$
\phi(\zeta)=\sqrt{\left(a^{2}-\zeta^{2}\right)\left(\zeta^{2}-b^{2}\right)} .
$$

For $|\zeta| \rightarrow \infty, \phi(\zeta) \rightarrow \mp i \zeta^{2}$, where the upper (lower) sign holds in the upper (lower) half plane. Just above (below) the real axis,

$$
\phi(x \pm i \epsilon)= \begin{cases} \pm i \tilde{\phi}(x), & |x|<b \text { or }|x|>a, \\ \tilde{\phi}(x), & b<|x|<a\end{cases}
$$

where

$$
\tilde{\phi}(x)= \begin{cases}\sqrt{\left(a^{2}-x^{2}\right)\left(b^{2}-x^{2}\right)}, & |x|<b, \\ \operatorname{sgn}(x) \sqrt{\left(a^{2}-x^{2}\right)\left(x^{2}-b^{2}\right)}, & b<|x|<a, \\ -\sqrt{\left(x^{2}-a^{2}\right)\left(x^{2}-b^{2}\right)}, & |x|>a .\end{cases}
$$

As can be seen from Eqs. (11) and (B2), $F(\zeta)$ is an analytic function of $\zeta=x+i y$ except for poles at $\zeta= \pm i y_{1}$ and $\zeta= \pm i y_{2}$, and a branch cut along the real axis.

Next consider the following integral around the closed contour $C$ consisting of a line just above the real axis at $\zeta^{\prime}=u+i \epsilon$ from $u=-\infty$ to $u=+\infty$, the infinite circle at $\zeta^{\prime}=R e^{i \theta}$ from $\theta=0$ to $\theta=2 \pi$ with $R \rightarrow \infty$, and a line just below the real axis at $\zeta^{\prime}=u-i \epsilon$ from $u=+\infty$ to $u=-\infty$,

$$
\oint_{C} d \zeta^{\prime} \frac{F\left(\zeta^{\prime}\right)}{\zeta^{\prime}-\zeta}=\int_{-\infty}^{+\infty} d u \frac{F(u+i \epsilon)-F(u-i \epsilon)}{u-\zeta},
$$

where the integral around the infinite circle vanishes because $|F(\zeta)| \propto\left|H(\zeta) / \zeta^{2}\right| \rightarrow 0$ as $|\zeta| \rightarrow \infty$. Using the residue theorem, accounting for the poles of the integrand 
on the left-hand side of Eq. (B66) at $\zeta^{\prime}=\zeta, i y_{1}, i y_{2},-i y_{1}$, and $-i y_{2}$, we obtain

$$
F(\zeta)-F_{0}(\zeta)=\frac{1}{2 \pi i} \int_{-\infty}^{+\infty} d u \frac{F(u+i \epsilon)-F(u-i \epsilon)}{u-\zeta},
$$

where

$$
\begin{aligned}
F_{0}(\zeta) & =\frac{I_{0}}{2 \pi}\left(\frac{y_{1}}{s_{1}\left(\zeta^{2}+y_{1}^{2}\right)}-\frac{y_{2}}{s_{2}\left(\zeta^{2}+y_{2}^{2}\right)}\right), \\
s_{1} & =\sqrt{\left(a^{2}+y_{1}^{2}\right)\left(b^{2}+y_{1}^{2}\right)}, \\
s_{2} & =\sqrt{\left(a^{2}+y_{2}^{2}\right)\left(b^{2}+y_{2}^{2}\right)} .
\end{aligned}
$$

From Eqs. (11), (2), and (B2), we find that

$$
\mathcal{H}(x \pm i \epsilon)-\mathcal{H}_{\|}(x \pm i \epsilon)=H_{y}(x, 0) \mp i K_{z}(x) / 2,
$$

such that Eqs. (B1) and (B4) yield

$F(u+i \epsilon)-F(u-i \epsilon)= \begin{cases}-2 i H_{y}(x, 0) / \tilde{\phi}(x), & |x|<b, \\ -i K_{z}(x) / \tilde{\phi}(x), & b<|x|<a, \\ -2 i H_{y}(x, 0) / \tilde{\phi}(x), & |x|>a .\end{cases}$

However, $H_{y}(x, 0)=0$ for $|x|<b$ or $|x|>a$, and $K_{z}(x)=-K_{c}$ for $b<|x|<a$. Using Eqs. (BB5) and (B12) and evaluating the integrals, we obtain the following expressions for the complex field $\mathcal{H}(\zeta)$ and complex potential $\mathcal{G}(\zeta)=\int_{i \epsilon}^{\zeta} \mathcal{H}\left(\zeta^{\prime}\right) d \zeta^{\prime}$ :

$$
\begin{aligned}
\mathcal{H}(\zeta)= & \frac{I_{0}}{2 \pi}\left\{\frac{y_{1}\left[i+\phi(\zeta) / s_{1}\right]}{\zeta^{2}+y_{1}^{2}}-\frac{y_{2}\left[i+\phi(\zeta) / s_{2}\right]}{\zeta^{2}+y_{2}^{2}}\right\} \\
& \pm i K_{c} / 2, \\
\mathcal{G}(\zeta)= & \frac{I_{0}}{2 \pi}\left[g_{0}\left(\zeta, y_{1}\right)-g_{0}\left(\zeta, y_{2}\right)\right] \pm i \frac{K_{c}}{2} \zeta,
\end{aligned}
$$

where

$$
\begin{aligned}
g_{0}(\zeta, y)= & \int_{ \pm i \epsilon}^{\zeta} d \zeta^{\prime} \frac{y\left[i+\phi\left(\zeta^{\prime}\right) / s\right]}{\zeta^{\prime 2}+y^{2}} \\
= & i \arctan (\zeta / y) \\
& \mp \frac{i}{a s y}\left[a^{2} y^{2} \boldsymbol{E}(\theta, k)+y^{2}\left(b^{2}+y^{2}\right) \boldsymbol{F}(\theta, k)\right. \\
& \left.-\left(a^{2}+y^{2}\right)\left(b^{2}+y^{2}\right) \boldsymbol{\Pi}\left(\theta,-b^{2} / y^{2}, k\right)\right],(\mathrm{B} 15) \\
s= & \sqrt{\left(a^{2}+y^{2}\right)\left(b^{2}+y^{2}\right),} \\
\theta= & \arcsin (\zeta / b), \text { and } \\
k= & b / a,
\end{aligned}
$$

where $\boldsymbol{E}, \boldsymbol{K}$, and $\boldsymbol{\Pi}$ are incomplete elliptic integrals.

When $b=0$, the following replacements can be made in the above expressions,

$$
\begin{aligned}
\phi(\zeta) & =\zeta \sqrt{a^{2}-\zeta^{2}}, \\
s_{1} & =y_{1} \sqrt{a^{2}+y_{1}^{2}}, \\
s_{2} & =y_{2} \sqrt{a^{2}+y_{2}^{2}},
\end{aligned}
$$

and $\boldsymbol{E}, \boldsymbol{K}$, and $\boldsymbol{\Pi}$ can be evaluated to obtain

$$
\begin{aligned}
g_{0}(\zeta, y) & =i \arctan (\zeta / y)+\sqrt{\frac{a^{2}-\zeta^{2}}{a^{2}+y^{2}}}-\frac{a}{\sqrt{a^{2}+y^{2}}} \\
& -\operatorname{arctanh} \sqrt{\frac{a^{2}-\zeta^{2}}{a^{2}+y^{2}}}+\operatorname{arctanh} \frac{a}{\sqrt{a^{2}+y^{2}}} .
\end{aligned}
$$

It follows from Eq. (11) that the integral $\int \mathcal{H}(\zeta) d \zeta$ around the great circle at $|\zeta| \rightarrow \infty$ yields $i \int K_{z}(x) d x$ along the real axis. The requirement that the film carries no net current is thus equivalent to the requirement that $\int \mathcal{H}(\zeta) d \zeta=0$. Using Eq. (B13) and the property that for $|\zeta| \rightarrow \infty, \phi(\zeta) \rightarrow \mp i \zeta^{2}$, where the upper (lower) sign holds in the upper (lower) half plane, we obtain the requirement that when $K_{c}=0, y_{1} / s_{1}=y_{2} / s_{2}$. Solving the latter equation, we obtain the following condition relating $a$ and $b$,

$$
a b=x_{0}^{2}=y_{1} y_{2} .
$$

Similarly, when $K_{c}>0$ and $b>0$, the requirement that the film carries no net current leads to the condition that

$$
\frac{I_{0}}{\pi}\left(\frac{y_{1}}{\sqrt{\left(a^{2}+y_{1}^{2}\right)\left(b^{2}+y_{1}^{2}\right)}}-\frac{y_{2}}{\sqrt{\left(a^{2}+y_{2}^{2}\right)\left(b^{2}+y_{2}^{2}\right)}}\right)=K_{c} .
$$

When $K_{c}>0$ but $b=0$, the same requirement leads to the condition that

$$
\frac{I_{0}}{\pi}\left(\frac{1}{\sqrt{a^{2}+y_{1}^{2}}}-\frac{1}{\sqrt{a^{2}+y_{2}^{2}}}\right)=K_{c} .
$$

1 W. DeSorbo and W. A. Healy, Cryogenics 4, 257 (1964).

2 D. C. Baird, Can. J. Phys. 42, 1682 (1964).

${ }^{3}$ F. Haenssler and L. Rinderer, Helv. Phys. Acta 40, 659 (1967).

4 R. P. Huebener, R. T. Kampwirth, and J. R. Clem, J. Low Temp. Phys. 6, 275 (1972).
5 J. R. Clem, R. P. Huebener, and D. E. Gallus, J. Low Temp. Phys. 12, 449 (1973).

6 J. Provost, E. Paumier, and A. Fortini, J. Phys. F 4, 439 (1974).

7 A. Fortini and E. Paumier, Phys. Rev. B 14, 55 (1976).

8 A. Fortini, A. Hairie, and E. Paumier, Phys. Rev. B 21, 
5065 (1980).

9 H. Castro, B. Dutoit, A. Jacquier, M. Baharami, and L. Rinderer, Phys. Rev. B 59, 596 (1999).

10 M. V. Indenbom, H. Kronmüller, T. W. Li, P. H. Kes and A. A. Menovsky, Physica C 222, 203 (1994).

11 Th. Schuster, M. V. Indenbom, H. Kuhn, E. H. Brandt, M. Konczykowski, Phys. Rev. Lett. 731424 (1994).

12 E. Zeldov, A. I. Larkin, V. B. Geshkenbein, M. Konczykowski, D. Majer, B. Khaykovich, V. M. Vinokur, and H. Shtrikman, Phys. Rev. Lett. 73, 1428 (1994).

13 I. L. Maksimov and A. A. Elistratov, Pis'ma Zh. Eksp. Teor. Fiz. 61, 204 (1995) [JETP Lett. 32, 753 (1995)].

14 I. L. Maksimov, Europhys. Lett. 32, 753 (1995).

15 N. Morozov, E. Zeldov, D. Majer, B. Khaykovich, Phys. Rev. Lett. 76, 138 (1996).

16 M. Benkraouda and J. R. Clem, Phys. Rev. B 53, 5716 (1996).

17 T. B. Doyle, R. Labusch, and R. A. Doyle, Physica C 290, 148 (1997).

18 E. H. Brandt, Phys. Rev. B 59, 3369 (1999).

19 E. H. Brandt, Phys. Rev. B 60, 11939 (1999).

20 Y. Mawatari and J. R. Clem, Phys. Rev. B 68, 24505 (2003).

21 C. P. Bean, Phys. Rev. Lett. 8, 250 (1962); Rev. Mod. Phys. 36, 31 (1964).
22 Because of the Bean-Livingston barrier [C. P. Bean and J. D. Livingston, Phys. Rev. Lett. 12, 14 (1973)], vortexantivortex penetration could be delayed until the field parallel to the surface reaches a value $H_{s}$, higher than $H_{c 1}$. If this effect occurs, $H_{c 1}$ in this paper should be replaced by $H_{s}$.

23 A. M. Campbell and J. E. Evetts, Adv. Phys. 21, 199 (1972).

24 J. R. Clem, J. Appl. Phys. 50, 3518 (1979).

25 Y. Mawatari and J. R. Clem (unpublished).

26 J. H. Claassen, M. E. Reeves, and R. J. Soulen, Jr., Rev. Sci. Instrum. 62, 996 (1991).

27 G. D. Poulin, J. S. Preston, and T. Strach, Phys. Rev. B 48, 1077 (1993).

28 Y. Mawatari, H. Yamasaki, and Y. Nakagawa, Appl. Phys. Lett. 81, 2424 (2002).

29 H. Yamasaki, Y. Mawatari, and Y. Nakagawa, Appl. Phys. Lett. 82, 3275 (2003).

30 H. Hochmuth and M. Lorenz, Physica C 220, 209 (1994); ibid. 265, 335 (1996).

31 J. R. Clem and M. W. Coffey, Phys. Rev. B 46, 14662 (1992).

32 J. Pearl, Appl. Phys. Lett. 5, 65 (1964). 